\title{
Global Well-Posedness for Models of Shallow Water in a Basin with a Varying Bottom
}

\author{
C. David Levermore, Marcel Oliver, 8 Edriss S. Titi
}

\begin{abstract}
We prove global well-posedness for the great lake equations. These equations arise to first order in a low aspect ratio, low Froude number (i.e. low wave speed) and very small wave amplitude expansion of the three dimensional incompressible Euler equations in a basin with a free upper surface and a spatially varying bottom topography.

On an abstract level, we consider a system that generalizes the two dimensional Euler equations in the following sense: while in the Euler system the vorticity field is given as the curl of the velocity field, here the two fields are related by a general linear operator enjoying analogous regularity properties. Moreover, the problem is posed in Sobolev spaces with a nondegenerate weight. In this setting, we follow the approach of Yudovitch and Bardos in constructing the solutions as the inviscid limit of solutions to a system with artificial viscosity which is the analog of the Navier-Stokes with respect to the Euler equations. The continuous dependence of the solutions on the initial data and the weight function is shown by a modification of the uniqueness estimate.
\end{abstract}

1. Introduction. We consider the following abstract setting for a family of shallow water equations that model the circulation of an inviscid fluid in a large shallow basin with a varying bottom. The evolution of the scalar potential vorticity $\omega=\omega(\boldsymbol{x}, t)$ and the corresponding vertically averaged velocity $\boldsymbol{u}=\boldsymbol{u}(\boldsymbol{x}, t)$ 
shall be described by a system of the form

$$
\begin{gathered}
\partial_{t} \omega+\boldsymbol{u} \cdot \nabla \omega=0, \\
\boldsymbol{u}=K \omega, \\
\omega(0)=\omega^{\text {in }} .
\end{gathered}
$$

The horizontal position coordinate $\boldsymbol{x}$ ranges over a bounded domain $\Omega \subset \mathbb{R}^{2}$ whose closure is denoted $\bar{\Omega}$. The linear operator $K$ is required to be such that $\boldsymbol{u}$ satisfies the weighted incompressibility condition

$$
\boldsymbol{\nabla} \cdot(b \boldsymbol{u})=0,
$$

where the weight $b=b(\boldsymbol{x})$ physically describes the depth of the basin at location $\boldsymbol{x}$. This system generalizes the two dimensional Euler equations in the following sense: whereas $\omega=\boldsymbol{\nabla} \wedge \boldsymbol{u} \equiv \partial_{1} u_{2}-\partial_{2} u_{1}$ for the Euler system, the relation between the velocity and vorticity fields is now prescribed by a linear operator $K$, which can be viewed as a perturbation of the inverse of the curl operator endowed with appropriate boundary conditions, and is assumed to have similar smoothing properties.

Mathematically, condition (1.2) makes $b(\boldsymbol{x}) \mathrm{d}^{2} \boldsymbol{x}$ the invariant measure of the Lagrangian flow $\dot{\boldsymbol{x}}=\boldsymbol{u}$, where $\mathrm{d}^{2} \boldsymbol{x}$ denotes the two dimensional Lebesgue measure. Hence it is natural to pose (1.1) in Sobolev spaces with weight $b$, i.e. to endow $\mathrm{H}^{n}(\Omega)$ for $n \in \mathbb{N}$ with the scalar product

$$
(\varphi, \vartheta)_{\mathrm{H}^{n}}=\sum_{0 \leq|\alpha| \leq n} \int_{\Omega} D^{\alpha} \varphi(\boldsymbol{x}) D^{\alpha} \vartheta(\boldsymbol{x}) b(\boldsymbol{x}) \mathrm{d}^{2} \boldsymbol{x} .
$$

Our main assumption is that the bottom function $b$ is nondegenerate, i.e. that there are constants $b_{\min }$ and $b_{\max }$ such that for every $\boldsymbol{x} \in \Omega$,

$$
0<b_{\min } \leq b(\boldsymbol{x}) \leq b_{\max } .
$$

This nondegeneracy means that the norm associated with (1.3) is equivalent to the canonical norm on $\mathrm{H}^{n}(\Omega)$, i.e. the weight $b$ affects the geometry, but not the topology. The degenerate case, namely $b(\boldsymbol{x})=0$ on $\partial \Omega$ or part of $\partial \Omega$ causes additional complications and will be studied in subsequent work.

The weighted integral over the domain is abbreviated by $\langle\cdot\rangle$, i.e.

$$
\langle\varphi\rangle=\int_{\Omega} \varphi(\boldsymbol{x}) b(\boldsymbol{x}) \mathrm{d}^{2} \boldsymbol{x},
$$

so that the $\mathrm{L}^{2}(\Omega)$ scalar product can be written $(\varphi, \vartheta)_{\mathrm{L}^{2}} \equiv\langle\varphi \vartheta\rangle$. Similarly, the Lebesgue spaces $\mathrm{L}^{p}(\Omega)$ and the Sobolev spaces $\mathrm{W}^{n, p}(\Omega)$ - the spaces of functions whose derivatives up to order $n$ are in $\mathrm{L}^{p}$-are defined with respect to the $b$ weighted integral. The nondegeneracy of $b$ again assures us that the weighted 
norms are equivalent to the norms in the unweighted case, so that all well-known embeddings as can be found in [1] still hold. Finally, we introduce the space of infinitely differentiable and compactly supported functions which satisfy our weighted incompressibility condition,

$$
\mathbb{D}=\left\{\boldsymbol{u} \in \mathrm{C}_{0}^{\infty}\left(\Omega, \mathbb{R}^{2}\right): \nabla \cdot(b \boldsymbol{u})=0 \text { in } \Omega\right\},
$$

and denote by $\mathbb{H}$ and $\mathbb{V}$ its completions in the $\mathrm{L}^{2}$ and $\mathrm{H}^{1}$ norm respectively. The $\mathrm{L}^{2}$-orthogonal (with respect to the $b$-weighted scalar product) projector onto $\mathbb{H}$ shall be denoted by $P$, and $Q \equiv 1-P$. Properties of these spaces in the unweighted case can be found in $[6,9,16]$.

The main objective of this paper then is to prove the following.

Theorem 1 Given a bounded domain $\Omega$ with $\partial \Omega \in \mathrm{C}^{2}$, a nondegenerate weight function $b \in \mathrm{C}^{2}(\bar{\Omega})$ and a continuous linear operator $K: \mathrm{L}^{2}(\Omega) \rightarrow \mathbb{V}$ which for some some $p_{0}>1$ satisfies the estimate

$$
\|K \omega\|_{\mathrm{W}^{1, p}} \leq c p\|\omega\|_{\mathrm{L}^{p}}
$$

for every $p \geq p_{0}$ where the constant $c$ may depend only on $p_{0}$ and $\Omega$, and given initial data $\omega^{\text {in }} \in \mathrm{L}^{\infty}(\Omega)$, there exists a unique function

$$
\omega \in \mathrm{C}\left([0, \infty) ; \mathrm{w}^{*}-\mathrm{L}^{\infty}([0, \infty) \times \Omega)\right) \cap \mathrm{L}^{\infty}([0, \infty) \times \Omega)
$$

that satisfies the weak form of the system (1.1), given by

$$
\begin{gathered}
\left\langle\psi \omega\left(t_{2}\right)\right\rangle-\left\langle\psi \omega\left(t_{1}\right)\right\rangle-\int_{t_{1}}^{t_{2}}\langle(\boldsymbol{u} \cdot \nabla \psi) \omega\rangle \mathrm{d} t=0, \\
\boldsymbol{u}=K \omega, \\
\omega(0)=\omega^{\text {in }},
\end{gathered}
$$

for every $\left[t_{1}, t_{2}\right] \subset[0, \infty)$ and every test function $\psi \in \mathrm{H}_{0}^{1}(\Omega)$.

We will also prove for a concrete choice of $K$ that the solution $\omega$ depends continuously on the initial data and the weight function $b$. It is clear from condition (1.2) that $K$ must also depend on $b$, hence there is no natural abstract continuity result. We will also establish existence theorems for spaces of initial data larger than $\mathrm{L}^{\infty}(\Omega)$, but $\mathrm{L}^{\infty}(\Omega)$ is the largest space for which we have established uniqueness. This is natural in the sense that the potential vorticity $\omega$ is a fundamental kinematic quantity [18].

Equations (1.1) arise in the study of an incompressible and inviscid fluid contained in a shallow basin with vertical lateral boundaries in a uniform gravitational field. The full dynamics is thus described by the Euler equations in three spatial dimensions. We consider the regime where the aspect ratio $\delta$ (the ratio of vertical to horizontal length scales) and the Froude number $\varepsilon$ (the ratio of typical 
horizontal speeds to gravity wave speeds) are small, and the wave amplitude is very small. In this limit, the evolution of the vertically averaged velocity vector $\boldsymbol{u}(\boldsymbol{x}, t)$ is governed to first order in $\delta^{2}$ by the set of nondimensional equations

$$
\begin{gathered}
\partial_{t} \boldsymbol{v}-\boldsymbol{u}^{\perp} \boldsymbol{\nabla} \wedge \boldsymbol{v}+\boldsymbol{\nabla}\left(h-\frac{1}{2} \boldsymbol{u} \cdot \boldsymbol{u}+\boldsymbol{u} \cdot \boldsymbol{v}\right)=0 \quad \text { in } \Omega \\
\boldsymbol{v}=\mathcal{L} \boldsymbol{u} \quad \text { in } \Omega, \\
\boldsymbol{\nabla} \cdot(b \boldsymbol{u})=0 \quad \text { in } \Omega, \\
\boldsymbol{u} \cdot \boldsymbol{n}=0 \quad \text { on } \partial \Omega \\
\boldsymbol{u}(0)=\boldsymbol{u}^{\text {in }} .
\end{gathered}
$$

Here $h(\boldsymbol{x}, t)$ denotes the surface height variation, $b(\boldsymbol{x})$ the depth of the basin, $\boldsymbol{n}(\boldsymbol{x})$ a unit vector normal on $\partial \Omega$, and we use the notation $\boldsymbol{u}^{\perp}=\left(u_{2},-u_{1}\right)$ and $\boldsymbol{\nabla} \wedge \boldsymbol{v}=\partial_{1} v_{2}-\partial_{2} v_{1}$. The linear operator $\mathcal{L}$ is given by

$$
\begin{aligned}
\boldsymbol{v}=\mathcal{L} \boldsymbol{u}=\boldsymbol{u}+\delta^{2} & \left((\boldsymbol{u} \cdot \boldsymbol{\nabla} b) \boldsymbol{\nabla} b+\frac{1}{2} b(\boldsymbol{\nabla} \cdot \boldsymbol{u}) \boldsymbol{\nabla} b\right. \\
& \left.-\frac{1}{2} b^{-1} \boldsymbol{\nabla}\left(b^{2} \boldsymbol{u} \cdot \boldsymbol{\nabla} b\right)-\frac{1}{3} b^{-1} \boldsymbol{\nabla}\left(b^{3} \boldsymbol{\nabla} \cdot \boldsymbol{u}\right)\right) .
\end{aligned}
$$

This system is called the great lake equations and has been derived in $[5,6]$. The zeroth or leading order approximation, called the lake equations, can be obtained by letting $\delta^{2}=0$, so that $\boldsymbol{v}=\boldsymbol{u}$. Another derivation of the lake equations can be found in [13].

It is easily verified that for a flat bottom both the lake and the great lake equations formally reduce to the two dimensional Euler equations. Moreover, in references $[5,6]$, it was shown that these shallow water models have a Hamiltonian structure, convect potential vorticity and enjoy a Kelvin circulation theorem in complete analogy with the pure Euler case. We extend this analogy herein by showing that the great lake equations can be treated within the framework of Theorem 1, the proof of which is an abstraction of the viscosity techniques developed by Yudovitch [22] and Bardos [4] for proving existence and uniqueness of weak solutions to the two dimensional Euler equations. In forthcoming work we extend this analogy further by establishing global well-posedness for classical data [17] and analytic data [14].

The paper is laid out as follows. In the next section we will recast the great lake equations into the form (1.1). The derivation of estimate (1.7) will require slightly stronger regularity assumptions on $b$ and $\Omega$ than the statement of the the purely abstract well-posedness result, Theorem 1 . The extensions necessary for treating non-simply connected domains are discussed in Section 3. The proof of existence and uniqueness of a solution is achieved in two steps. In Section 4 we will first consider the system with an artificial viscosity term, and in Section 5 show that one can pass to its inviscid limit. In Section 6 we will use the special properties of the great lake equations to prove continuous dependence of the solution on the initial data and on the bottom topography. Altogether these results imply that the great lake equations are well posed. 
2. The vorticity formulation. The goal of this section is to derive the vorticity formulation (1.1) from the great lake equations (1.10), and to prove that the resulting operator $K$ indeed possesses the properties required by the abstract existence and uniqueness result.

By taking the curl of the momentum equation (1.10a), using an identity which is based on the fact that $\boldsymbol{\nabla} \cdot(b \boldsymbol{u})=0$,

$$
\begin{aligned}
(\boldsymbol{u} \cdot \boldsymbol{\nabla})\left(b^{-1} \boldsymbol{\nabla} \wedge \boldsymbol{v}\right) & =-b^{-2}(\boldsymbol{u} \cdot \boldsymbol{\nabla} b) \boldsymbol{\nabla} \wedge \boldsymbol{v}+b^{-1}(\boldsymbol{u} \cdot \boldsymbol{\nabla}) \boldsymbol{\nabla} \wedge \boldsymbol{v} \\
& =b^{-1}(\boldsymbol{\nabla} \cdot \boldsymbol{u}+\boldsymbol{u} \cdot \boldsymbol{\nabla})(\boldsymbol{\nabla} \wedge \boldsymbol{v}) \\
& =-b^{-1} \boldsymbol{\nabla} \wedge\left(\boldsymbol{u}^{\perp} \boldsymbol{\nabla} \wedge \boldsymbol{v}\right)
\end{aligned}
$$

and introducing the potential vorticity $\omega=b^{-1} \nabla \wedge \boldsymbol{v}$, we obtain

$$
\partial_{t} \omega+\boldsymbol{u} \cdot \nabla \omega=0 .
$$

Next, in order to recover relationship (1.1b) from the great lake equations, we introduce the stream function $\psi$ as the solution of the - as we will show below-elliptic problem

$$
\begin{gathered}
\omega=b^{-1} \nabla \wedge \mathcal{L}\left(b^{-1} \nabla^{\perp} \psi\right) \quad \text { in } \Omega, \\
\psi=0 \quad \text { on } \partial \Omega .
\end{gathered}
$$

The notation used here is $\nabla^{\perp}=\left(\partial_{2},-\partial_{1}\right)$, and for now we assume that $\Omega$ is simply connected. In the multiply connected case which is discussed in the next section, one has to specify different boundary values on each connected component of $\partial \Omega$. Then $\boldsymbol{u} \equiv K \omega$ is given by

$$
\boldsymbol{u}=b^{-1} \nabla^{\perp} \psi .
$$

Let us now investigate the smoothing properties of $K$ and verify that it imposes the correct boundary and incompressibility conditions on $\boldsymbol{u}$. In a preliminary step we shall investigate the operator $\mathcal{L}$ by itself.

Lemma 2 For an arbitrary bounded domain with $\partial \Omega \in \mathrm{C}^{1}$ and $b \in \mathrm{C}^{1}(\bar{\Omega})$, the restriction of $\mathcal{L}$ to the space $\mathbb{H}$ is a continuous, invertible, positive and selfadjoint operator, explicitly given by

$$
P \mathcal{L} P \boldsymbol{u}=P\left(\boldsymbol{u}+\frac{\delta^{2}}{3} \nabla b(\boldsymbol{\nabla} b \cdot P \boldsymbol{u})\right),
$$

for every $\boldsymbol{u} \in \mathrm{L}^{2}\left(\Omega, \mathbb{R}^{2}\right)$.

Remark 1 This lemma is useful because the null space of the projector $P$ is contained in the nullspace of the curl operator, and we therefore can write (2.3) as

$$
\begin{gathered}
\omega=b^{-1} \nabla \wedge P \mathcal{L} P\left(b^{-1} \nabla^{\perp} \psi\right) \quad \text { in } \Omega, \\
\psi=0 \quad \text { on } \partial \Omega .
\end{gathered}
$$


Proof. Assume first that $\boldsymbol{u}, \boldsymbol{v} \in \mathbb{D}$. This implies that $\boldsymbol{\nabla} \cdot \boldsymbol{u}=-b^{-1} \boldsymbol{u} \cdot \boldsymbol{\nabla} b$ the same being true for $\boldsymbol{v}$ - so that integration by parts and use of the boundary conditions on $\boldsymbol{u}$ and $\boldsymbol{v}$ gives

$$
\begin{aligned}
\ell(\boldsymbol{u}, \boldsymbol{v}) \equiv & (\mathcal{L} \boldsymbol{u}, \boldsymbol{v})_{\mathrm{L}^{2}} \\
= & (\boldsymbol{u}, \boldsymbol{v})_{\mathrm{L}^{2}}+\delta^{2} \int_{\Omega}\left(b(\boldsymbol{u} \cdot \boldsymbol{\nabla} b) \boldsymbol{\nabla} b+\frac{1}{2} b^{2}(\boldsymbol{\nabla} \cdot \boldsymbol{u}) \boldsymbol{\nabla} b\right. \\
& \left.\quad-\frac{1}{2} \boldsymbol{\nabla}\left(b^{2} \boldsymbol{u} \cdot \boldsymbol{\nabla} b\right)-\frac{1}{3} \boldsymbol{\nabla}\left(b^{3} \boldsymbol{\nabla} \cdot \boldsymbol{u}\right)\right) \cdot \boldsymbol{v} \mathrm{d}^{2} \boldsymbol{x} \\
= & (\boldsymbol{u}, \boldsymbol{v})_{\mathrm{L}^{2}}+\delta^{2} \int_{\Omega}\left(b(\boldsymbol{u} \cdot \boldsymbol{\nabla} b)(\boldsymbol{v} \cdot \boldsymbol{\nabla} b)+\frac{1}{2} b^{2}(\boldsymbol{\nabla} \cdot \boldsymbol{u})(\boldsymbol{v} \cdot \boldsymbol{\nabla} b)\right. \\
\quad & \left.\quad+\frac{1}{2} b^{2}(\boldsymbol{u} \cdot \boldsymbol{\nabla} b)(\boldsymbol{\nabla} \cdot \boldsymbol{v})+\frac{1}{3} b^{3}(\boldsymbol{\nabla} \cdot \boldsymbol{u})(\boldsymbol{\nabla} \cdot \boldsymbol{v})\right) \mathrm{d}^{2} \boldsymbol{x} \\
= & (\boldsymbol{u}, \boldsymbol{v})_{\mathrm{L}^{2}}+\frac{1}{3} \delta^{2}(\boldsymbol{u} \cdot \boldsymbol{\nabla} b, \boldsymbol{v} \cdot \boldsymbol{\nabla} b)_{\mathrm{L}^{2}} \\
\leq & c_{1}\|\boldsymbol{u}\|_{\mathrm{L}^{2}}\|\boldsymbol{v}\|_{\mathrm{L}^{2}}
\end{aligned}
$$

This shows that $\ell$ can be extended to a continuous, coercive and symmetric bilinear form on $\mathbb{H}$. Unique invertibility follows from the Lax-Milgram theorem and the explicit form of $\mathcal{L}$ can be directly read off equation (2.7) .

This leads to our main result on $K$.

Lemma 3 For a bounded and simply connected domain with $\partial \Omega \in \mathrm{C}^{3}$, $b \in \mathrm{C}^{3}(\bar{\Omega})$ and every $\omega \in \mathrm{H}^{-1}(\Omega)$ there exists a unique function $\boldsymbol{u}=K \omega \in$ $\mathbb{H}$. Moreover, $K$ is continuous as a mapping between the spaces $\mathrm{H}^{-1}(\Omega) \rightarrow \mathbb{H}$, $\mathrm{L}^{2}(\Omega) \rightarrow \mathbb{V}$ and $\mathrm{H}^{1}(\Omega) \rightarrow \mathbb{V} \cap \mathrm{H}^{2}\left(\Omega, \mathbb{R}^{2}\right)$, and for some $p_{0}>1$ satisfies the estimate

$$
\|K \omega\|_{\mathrm{W}^{1, p}} \leq c p\|\omega\|_{\mathrm{L}^{p}},
$$

for all $p \geq p_{0}$ where the constant $c$ depends only on $p_{0}$ and $\Omega$.

Proof. Let $\varphi, \psi \in \mathrm{C}_{0}^{\infty}(\Omega)$ and define $\boldsymbol{u} \equiv b^{-1} \nabla^{\perp} \varphi$ and $\boldsymbol{v} \equiv b^{-1} \nabla^{\perp} \psi$. The fact that the classical functions $\boldsymbol{u}$ and $\boldsymbol{v}$ are also members of $\mathbb{H}$ can be verified with a direct calculation. Now integrate by parts and use Lemma 2 to obtain

$$
\begin{aligned}
& \left(\varphi, b^{-1} \nabla \wedge \mathcal{L}\left(b^{-1} \nabla^{\perp} \psi\right)\right)_{L^{2}} \\
= & \int_{\Omega}\left(b^{-1} \nabla^{\perp} \varphi\right) \cdot \mathcal{L}\left(b^{-1} \nabla^{\perp} \psi\right) b \mathrm{~d}^{2} \boldsymbol{x} \leq c_{2}\|\boldsymbol{u}\|_{\mathrm{L}^{2}}\|\boldsymbol{v}\|_{\mathrm{L}^{2}} .
\end{aligned}
$$

Due to the Poincaré inequality and the nondegeneracy of $b,\left\|b^{-1} \nabla^{\perp} \cdot\right\|_{L^{2}}$ is an equivalent norm on $\mathrm{H}_{0}^{1}(\Omega)$, so that we can bound the left side of (2.9) from above and below by

$$
\left(\varphi, b^{-1} \nabla \wedge \mathcal{L}\left(b^{-1} \nabla^{\perp} \psi\right)\right)_{\mathrm{L}^{2}} \leq c_{3}\|\varphi\|_{\mathrm{H}^{1}}\|\psi\|_{\mathrm{H}^{1}}
$$

and 


$$
\|\varphi\|_{\mathrm{H}^{1}}^{2} \leq c_{4}\left(\varphi, b^{-1} \nabla \wedge \mathcal{L}\left(b^{-1} \nabla^{\perp} \varphi\right)\right)_{\mathrm{L}^{2}} .
$$

This proves continuity and coercivity of the bilinear form. We can now extend (2.10) to $\mathrm{H}_{0}^{1}(\Omega)$ functions by a density argument, so that the Lax-Milgram theorem implies unique solvability of problem $(2.3)$ for $\omega \in \mathrm{H}^{-1}(\Omega) \equiv \mathrm{H}_{0}^{1}(\Omega)^{\prime}$. By continuously extending the operator $b^{-1} \nabla^{\perp}$ to $\mathrm{H}_{0}^{1}(\Omega)$ functions, we can furthermore conclude that $\boldsymbol{u} \in \mathbb{H}$, and that $K: \mathrm{H}^{-1}(\Omega) \rightarrow \mathbb{H}$ is continuous.

Provided that $\omega \in \mathrm{L}^{p}(\Omega)$, well-known regularity theorems for elliptic equations allow us to conclude that $\psi \in \mathrm{W}^{2, p}(\Omega)$ and satisfies an estimate

$$
\|\psi\|_{\mathrm{W}^{2, p}} \leq c p\|\omega\|_{\mathrm{L}^{p}}
$$

where it is possible to choose the constant $c$ independent of $p$ as long as $p \geq p_{0}$ for some $p_{0}>1$, see references $[2,11,22]$. Estimate (2.8) follows directly.

In particular if $\omega \in \mathrm{L}^{2}(\Omega)$, then one can conclude that $\psi \in \mathrm{H}^{2}(\Omega) \cap \mathrm{H}_{0}^{1}(\Omega)$ which implies $\boldsymbol{u} \in \mathbb{V}$. Furthermore, if $\omega \in \mathrm{H}^{1}(\Omega)$, then $\psi \in \mathrm{H}^{3}(\Omega) \cap \mathrm{H}_{0}^{1}(\Omega)$ and $\boldsymbol{u} \in \mathrm{H}^{2}\left(\Omega, \mathbb{R}^{2}\right) \cap \mathbb{V}$. It is for this step that the regularity assumptions on $\partial \Omega$ and $b$ need to be as strong as stated, see $[11,21]$.

3. Multiply connected domains. When the domain $\Omega$ is non-simply connectedwhen the lake has islands - the velocity field ceases to be uniquely determined by the potential vorticity. Given $n$ islands $\Omega_{1}, \ldots, \Omega_{n}$, the missing parameters are the (constant) boundary values $\boldsymbol{\lambda}=\left(\lambda_{1}, \ldots, \lambda_{n}\right)$ of the stream function on each of the boundary components $\partial \Omega_{1}, \ldots, \partial \Omega_{n}$. Equivalently, we shall see that one can specify certain generalized circulations $\boldsymbol{\Gamma}=\left(\Gamma_{1}, \ldots, \Gamma_{n}\right)$ in order to uniquely determine $\boldsymbol{u}$. This is well known for classical inviscid fluid flow [9, 15, 22], and we will show here that these concepts naturally extend to the great lake equations (1.1) .

As before, we introduce a stream function $\psi$ and then compute the velocity field as $\boldsymbol{u}=b^{-1} \nabla^{\perp} \psi$. The boundary condition $\boldsymbol{u} \cdot \boldsymbol{n}=0$ on $\partial \Omega$ requires $\psi$ to be constant on each connected component $\partial \Omega_{i}$ of $\partial \Omega$. We can adopt the normalization $\psi=0$ on $\partial \Omega_{0}$, the outer boundary of $\Omega$, because $\boldsymbol{u}$ depends only on derivatives of the stream function. So the problem one has to solve is

$$
\begin{gathered}
\omega=b^{-1} \nabla \wedge P \mathcal{L} P\left(b^{-1} \nabla^{\perp} \psi\right) \quad \text { in } \Omega, \\
\psi=0 \quad \text { on } \partial \Omega_{0}, \\
\psi=\lambda_{i} \quad \text { on } \partial \Omega_{i},
\end{gathered}
$$

where $i=1, \ldots, n$. We first study the family of irrotational (homogeneous) problems

$$
\begin{gathered}
b^{-1} \nabla \wedge P \mathcal{L} P\left(b^{-1} \nabla^{\perp} \psi_{i}\right)=0 \quad \text { in } \Omega, \\
\left.\psi_{i}\right|_{\partial \Omega_{j}}=\delta_{i j} \quad \text { for } j=0, \ldots, n ; i \in 1, \ldots, n,
\end{gathered}
$$


which have associated velocity fields defined by

$$
\boldsymbol{u}_{i}=b^{-1} \nabla^{\perp} \psi_{i} .
$$

Naturally, one would expect the following.

Lemma 4 For $b \in \mathrm{C}^{3}(\bar{\Omega}), \partial \Omega \in \mathrm{C}^{3}$ and every $i \in 1, \ldots, n$ there exists a unique solution $\psi_{i} \in \mathrm{C}^{3}(\bar{\Omega})$ to problem (3.2). The set of associated velocities $\left\{\boldsymbol{u}_{i}\right\}$ is linearly independent. Moreover, a function

$$
\boldsymbol{u}=\sum_{i=1}^{n} \lambda_{i} \boldsymbol{u}_{i}
$$

is uniquely determined by the circulations

$$
\Gamma_{i}=\int_{\partial \Omega_{i}}(P \mathcal{L} P \boldsymbol{u}) \cdot \mathrm{d} \boldsymbol{l}
$$

where $i=1, \ldots, n$.

Proof. The operator on the left side of (3.2a) is strictly elliptic, as we have already shown in the proof of Lemma 3. Here we do not have to account for distributional data, so that the existence of a unique solution $\psi_{i}$ is readily guaranteed by the Schauder theory as can be found in [11].

We show the linear independence of $\left\{\boldsymbol{u}_{i}\right\}$ following Yudovitch [22]. Assume that

$$
\boldsymbol{u}=\sum_{i=1}^{n} \lambda_{i} \boldsymbol{u}_{i}=0
$$

Now compute the flux of the vector field $\boldsymbol{u}$ through some diaphragm $\gamma_{0 k}$, a curve connecting a point on $\partial \Omega_{0}$ with a point on $\partial \Omega_{k}$,

$$
0 \equiv \int_{\gamma_{0 k}} \boldsymbol{u} \cdot \boldsymbol{n} b \mathrm{~d} \ell=\sum_{i=1}^{n} \lambda_{i} \int_{\gamma_{0 k}} \boldsymbol{\nabla} \psi_{i} \cdot \boldsymbol{t} \mathrm{d} \ell=\sum_{i=1}^{n} \lambda_{i} \delta_{i k}=\lambda_{k} .
$$

This proves linear independence. We remark that the unit normal $\boldsymbol{n}$ on $\gamma_{0 k}$ and the unit tangent $\boldsymbol{t}$ in the direction from $\Omega_{0}$ to $\Omega_{k}$ are taken to form a right handed pair $(\boldsymbol{n}, \boldsymbol{t})$.

Lastly, uniqueness of $\boldsymbol{u}$ is proved using potential theory methods as employed by Marchioro and Pulvirenti [15] in the context of the Euler equations. We fix a point $\boldsymbol{x}_{0} \in \Omega$ and define a multivalued potential by

$$
U(\boldsymbol{x})=\int_{\gamma(\boldsymbol{x})}(P \mathcal{L} P \boldsymbol{u}) \cdot \mathrm{d} \boldsymbol{l},
$$


where $\gamma(\boldsymbol{x})$ is a path from $\boldsymbol{x}_{0}$ to some point $\boldsymbol{x} \in \Omega$. Lemma 2 and its proof do not depend on the connectivity of the domain. Moreover, the weighted Leray projection $P$ is continuous on $\mathrm{C}^{2}(\bar{\Omega})$, again a consequence of the Schauder theory for elliptic operators. We conclude that $P \mathcal{L} P$ is continuous and invertable on $\mathrm{C}^{2}(\bar{\Omega})$ so that the line integral in (3.8) can be interpreted in a classical sense.

The value of the line integral does not change under continuous deformations of the path. Thus we can insert $n$ diaphragms into the domain such that cuts along them will leave $\Omega$ simply connected. One can then deform $\gamma(\boldsymbol{x})$ such that (3.8) decomposes into a line integral along a path $\gamma^{\prime}(\boldsymbol{x})$ within this simply connected region, and some line integrals around the boundaries of the islands, i.e.

$$
U(\boldsymbol{x})=\int_{\gamma^{\prime}(\boldsymbol{x})}(P \mathcal{L} P \boldsymbol{u}) \cdot \mathrm{d} \boldsymbol{l}+\sum_{i=1}^{n} k_{i} \Gamma_{i},
$$

where $k_{i} \in \mathbb{Z}$. Now suppose that $\boldsymbol{v}=\overline{\boldsymbol{u}}-\hat{\boldsymbol{u}}$ denotes the difference of two solutions to (3.4) which have the same circulations $\boldsymbol{\Gamma}$. The associated potential is then a single-valued function

$$
V(\boldsymbol{x})=\int_{\gamma^{\prime}(\boldsymbol{x})}(P \mathcal{L} P \boldsymbol{v}) \cdot \mathrm{d} \boldsymbol{l} .
$$

By construction $V$ is harmonic and $\boldsymbol{n} \cdot \boldsymbol{\nabla} V=0$ on $\partial \Omega$, so $V$ is a constant. This implies uniqueness of $P \mathcal{L} P \boldsymbol{u}$, and hence uniqueness of $\boldsymbol{u}$.

Remark 2 The main assertion of Lemma 4 can be expressed as follows: There exists a nonsingular linear transformation $S$ such that $\boldsymbol{\lambda}=S \boldsymbol{\Gamma}$.

We can now obtain the general velocity field $\boldsymbol{u}$ associated with problem (3.1) as the sum of the general solution to the homogeneous problem, given by Lemma 4, and a particular solution to the inhomogeneous problem, guaranteed by Lemma 3 as the solvability assertion is not affected by assuming a multiply connected domain. Altogether we obtain a generalization of Lemma 3.

Lemma 5 Let $\Omega$ be a multiply connected domain with connectivity $n+1$ and $\partial \Omega \in \mathrm{C}^{3}$, and let $b \in \mathrm{C}^{3}(\bar{\Omega})$. Then for every tuple $(\omega, \boldsymbol{\Gamma})$ with $\omega \in \mathrm{H}^{-1}(\Omega)$ and $\boldsymbol{\Gamma}=\left(\Gamma_{1}, \ldots, \Gamma_{n}\right)$ there exists a unique function $\boldsymbol{u} \equiv K \omega \in \mathbb{H}$ which satisfies $\omega=b^{-1} \nabla \wedge P \mathcal{L} P \boldsymbol{u}$ and

$$
\Gamma_{i}=\int_{\partial \Omega_{i}}(P \mathcal{L} P \boldsymbol{u}) \cdot \mathrm{d} \boldsymbol{l}
$$

Moreover, $K$ is continuous as a mapping between the spaces $\mathrm{H}^{-1}(\Omega) \rightarrow \mathbb{H}$, $\mathrm{L}^{2}(\Omega) \rightarrow \mathbb{V}$ and $\mathrm{H}^{1}(\Omega) \rightarrow \mathbb{V} \cap \mathrm{H}^{2}\left(\Omega, \mathbb{R}^{2}\right)$, and for some $p_{0}>1$ satisfies the 
estimate

$$
\|K \omega\|_{\mathrm{W}^{1, p}} \leq c p\|\omega\|_{\mathrm{L}^{p}}+c^{\prime}|\boldsymbol{\Gamma}|,
$$

for every $p \geq p_{0}$ where the constants $c$ and $c^{\prime}$ depend only on $p_{0}$ and $\Omega$.

We finally remark that $\boldsymbol{\Gamma}$ is a constant of the motion for the great lake equations (1.10) because of the boundary condition $\boldsymbol{u} \cdot \boldsymbol{n}=0$ on $\partial \Omega$. Hence $\boldsymbol{\Gamma}$ does not appear explicitly in the remainder of this paper, although one should keep in mind that in the multiply connected case not only does $K$ depend on the geometry of the lake, but also on the initial velocity field.

4. Existence and uniqueness for an artificial viscosity system. In this section we will prove existence and uniqueness of solutions for a system with an artificial viscosity term. We first establish the functional setting and prove some identities that will be used frequently throughout the proofs. We will assume from now on that $\partial \Omega \in \mathrm{C}^{3}$ and $b \in \mathrm{C}^{3}(\bar{\Omega})$.

First, define an operator $A$ by

$$
\begin{gathered}
\omega=-b^{-1} \nabla \cdot(b \nabla \psi) \equiv A \psi \quad \text { on } \Omega, \\
\psi=0 \quad \text { on } \partial \Omega,
\end{gathered}
$$

i.e., $-A$ can be thought of as a Laplace operator on Sobolev spaces with weight $b$. Note that this definition does not depend on the connectivity of the domain. For $\omega \in \mathrm{C}_{0}^{\infty}(\Omega)$,

$$
\langle A \omega \varphi\rangle=\langle\nabla \omega \cdot \nabla \varphi\rangle \leq\|\omega\|_{\mathrm{H}^{1}}\|\varphi\|_{\mathrm{H}^{1}}
$$

which shows that $\langle\nabla \omega \cdot \nabla \varphi\rangle$ can be extended to a symmetric, continuous anddue to the Poincaré inequality - coercive bilinear form on $\mathrm{H}_{0}^{1}(\Omega)$, a closed subspace of $\mathrm{H}^{1}(\Omega)$. By the Lax-Milgram theorem, $A: \mathrm{H}_{0}^{1}(\Omega) \rightarrow \mathrm{H}_{0}^{1}(\Omega)^{\prime} \equiv \mathrm{H}^{-1}(\Omega)$ is an isomorphism. Its positivity allows us to define arbitrary powers of $A$, so that we can identify the $\mathrm{H}^{1}$ norm on $\mathrm{H}_{0}^{1}(\Omega)$ with $\left\|A^{1 / 2} \cdot\right\|_{\mathrm{L}^{2}}$ and the $\mathrm{H}^{2}$ norm on $\mathrm{H}^{2}(\Omega) \cap \mathrm{H}_{0}^{1}(\Omega)$ with $\|A \cdot\|_{\mathrm{L}^{2}}$. Similarly, $\mathrm{H}^{-1}(\Omega)=\mathrm{H}_{0}^{1}(\Omega)^{\prime}$ can be endowed with norm $\left\|A^{-1 / 2} \cdot\right\|_{\mathrm{L}^{2}}$ and scalar product $\left(A^{-1 / 2} \cdot, A^{-1 / 2} \cdot\right)_{\mathrm{L}^{2}}$.

For $\boldsymbol{u} \in \mathbb{D}$ and $\omega \in \mathrm{C}^{\infty}(\bar{\Omega})$ one establishes through integration by parts (i.e. the Gauss integral formula) that

$$
\begin{aligned}
\langle(\boldsymbol{u} \cdot \nabla \omega) \omega\rangle=\int_{\partial \Omega} \boldsymbol{n} \cdot \boldsymbol{u} \omega^{2} b \mathrm{~d} \sigma & \\
& -\int_{\Omega} \boldsymbol{\nabla} \cdot(b \boldsymbol{u}) \omega^{2} \mathrm{~d}^{2} \boldsymbol{x}-\langle(\boldsymbol{u} \cdot \boldsymbol{\nabla} \omega) \omega\rangle .
\end{aligned}
$$


The first two terms on the right side clearly vanish so that the remaining terms must vanish, too. This implies

$$
\langle(\boldsymbol{u} \cdot \nabla \omega) \varphi\rangle=-\langle(\boldsymbol{u} \cdot \nabla \varphi) \omega\rangle,
$$

for every $\boldsymbol{u} \in \mathbb{D}$ and $\omega, \varphi \in \mathrm{C}^{\infty}(\bar{\Omega})$. This relation can be extended by continuity to all $\boldsymbol{u} \in \mathbb{H}$ and $\omega, \varphi \in \mathrm{H}^{1}(\Omega)$.

Now we consider the following system which consists of the potential vorticity equation (1.1) with an extra viscosity term. In the next section we will then show how to pass to the inviscid $(\nu \rightarrow 0)$ limit.

$$
\begin{gathered}
\partial_{t} \omega+\nu A \omega+\boldsymbol{u} \cdot \nabla \omega=0 \quad \text { in } \Omega, \\
\boldsymbol{u}=K \omega \quad \text { in } \Omega, \\
\omega=0 \quad \text { on } \partial \Omega, \\
\omega(0)=\omega^{\text {in }} .
\end{gathered}
$$

We assume that $K$ is a linear operator, continuous as a mapping between the spaces $\mathrm{H}^{-1}(\Omega) \rightarrow \mathbb{H}, \mathrm{H}^{1}(\Omega) \rightarrow \mathrm{H}^{2}\left(\Omega, \mathbb{R}^{2}\right) \cap \mathbb{V}$ and $\mathrm{L}^{p}(\Omega) \rightarrow \mathrm{W}^{1, p}\left(\Omega, \mathbb{R}^{2}\right)$, and for some $p_{0}>1$ satisfies the estimate

$$
\|K \omega\|_{\mathrm{W}^{1, p}} \leq c p\|\omega\|_{\mathrm{L}^{p}}
$$

for every $p \geq p_{0}$ where the constant $c$ may depend only on $p_{0}$ and $\Omega$.

The following theorem then establishes global existence of weak solutions to the problem (4.5).

Theorem 6 Given $\omega^{\text {in }} \in \mathrm{L}^{2}(\Omega)$, there exists a unique function

$$
\omega \in \mathrm{C}\left([0, \infty) ; \mathrm{w}-\mathrm{L}^{2}(\Omega)\right) \cap \mathrm{L}_{\text {loc }}^{2}\left([0, \infty) ; \mathrm{H}_{0}^{1}(\Omega)\right)
$$

that satisfies the weak form of system (4.5) given by

$$
\begin{gathered}
\left\langle\psi \omega\left(t_{2}\right)\right\rangle-\left\langle\psi \omega\left(t_{1}\right)\right\rangle+\nu \int_{t_{1}}^{t_{2}}\langle\boldsymbol{\nabla} \psi \cdot \nabla \omega\rangle \mathrm{d} t-\int_{t_{1}}^{t_{2}}\langle(\boldsymbol{u} \cdot \nabla \psi) \omega\rangle \mathrm{d} t=0, \\
\boldsymbol{u}=K \omega, \\
\omega(0)=\omega^{\text {in }},
\end{gathered}
$$

for every $\left[t_{1}, t_{2}\right] \subset[0, \infty)$ and every test function $\psi \in \mathrm{H}_{0}^{1}(\Omega)$. The solution satisfies the "energy relation"

$$
\|\omega(t)\|_{L^{2}} \leq\left\|\omega^{\text {in }}\right\|_{L^{2}}
$$

for every $t \in[0, \infty)$. Moreover, if $\omega^{\text {in }} \in \mathrm{H}_{0}^{1}(\Omega)$ one has

$$
\omega \in \mathrm{C}\left([0, \infty) ; \mathrm{H}_{0}^{1}(\Omega)\right) \cap \mathrm{L}_{\mathrm{loc}}^{2}\left([0, \infty) ; \mathrm{H}^{2}(\Omega) \cap \mathrm{H}_{0}^{1}(\Omega)\right)
$$


and the "energy relation"

$$
\|\omega(t)\|_{\mathrm{L}^{p}} \leq\left\|\omega^{\mathrm{in}}\right\|_{\mathrm{L}^{p}}
$$

for every $t \in[0, \infty)$ and $2 \leq p<\infty$.

Remark 3 Physically, for $p=2$ equation (4.10) is an inequality for the potential enstrophy. Formally, however, it plays the role of the energy in Leray's theory of weak solutions to the Navier-Stokes equations.

Remark 4 As the $\mathrm{L}^{p}$ bound (4.10) holds uniformly over all $p \in[2, \infty)$, it also holds for $p=\infty$ provided the initial data is $\mathrm{L}^{\infty}$.

Proof. We will use an equicontinuity argument as has been applied to the complex Ginzburg-Landau equation in [8]. In doing so, we slightly deviate from the procedure that is commonly found in the literature on the Navier-Stokes equations $[7,19,20]$, although the main principles are analogous. The proof proceeds in nine distinct steps.

Step 1 Construct a family of approximate solutions $\omega_{n}$ that have a consistent weak form and energy relation. This can be achieved many ways, however it is technically most convenient to use a Galerkin truncation to finite dimensional eigenspaces of the operator $A$. Let $P_{n}$ denote the $\mathrm{L}^{2}$-orthogonal (with respect to the $b$-weighted scalar product) projection onto the span of the eigenvectors corresponding to the lowest $n$ eigenvalues of $A$. Now let $\omega_{n}=\omega_{n}(t)$ be the unique solution to the ordinary differential initial value problem

$$
\begin{gathered}
\partial_{t} \omega_{n}+\nu A \omega_{n}+P_{n}\left(\boldsymbol{u}_{n} \cdot \nabla \omega_{n}\right)=0, \\
\boldsymbol{u}_{n}=K \omega_{n}, \\
\omega_{n}(0)=\omega_{n}^{\text {in }} \equiv P_{n} \omega^{\text {in }} .
\end{gathered}
$$

The regularization of the initial data $\omega_{n}^{\text {in }}$ converges to $\omega^{\text {in }}$ in $\mathrm{L}^{2}(\Omega)$ and satisfies $\left\|\omega_{n}^{\text {in }}\right\|_{\mathrm{L}^{2}} \leq\left\|\omega^{\text {in }}\right\|_{\mathrm{L}^{2}}$. It can then be shown that the $\omega_{n}$ satisfy the energy relation

$$
\left\|\omega_{n}(t)\right\|_{\mathrm{L}^{2}} \leq\left\|\omega_{n}^{\mathrm{in}}\right\|_{\mathrm{L}^{2}} \leq\left\|\omega^{\mathrm{in}}\right\|_{\mathrm{L}^{2}},
$$

and the weak form of the regularized equation (4.12a),

$$
\begin{gathered}
\left\langle\psi \omega_{n}\left(t_{2}\right)\right\rangle-\left\langle\psi \omega_{n}\left(t_{1}\right)\right\rangle+\nu \int_{t_{1}}^{t_{2}}\left\langle\boldsymbol{\nabla} \psi \cdot \nabla \omega_{n}\right\rangle \mathrm{d} t \\
-\int_{t_{1}}^{t_{2}}\left\langle\left(\boldsymbol{u}_{n} \cdot \nabla \psi_{n}\right) \omega_{n}\right\rangle \mathrm{d} t=0,
\end{gathered}
$$

for every $\left[t_{1}, t_{2}\right] \subset[0, \infty)$ and $\psi \in \mathrm{H}_{0}^{1}(\Omega)$ with $\psi_{n} \equiv P_{n} \psi$. 
The nonlinearity in (4.12a) is a quadratic polynomial, hence locally Lipschitz, so that local existence of a regularized solution $\omega_{n}$ is guaranteed by the PicardLindelöf existence theorem applied to (4.12) posed on the finite dimensional space $P_{n} \mathrm{~L}^{2}(\Omega)$. The global estimate (4.13) then assures that $\omega_{n}$ is in fact a global solution. The argument is classical so that we omit all details.

Step 2 Show that the sequence $\left\{\omega_{n}\right\}$ is a relatively compact set in

$$
\mathrm{C}\left([0, \infty) ; \mathrm{w}-\mathrm{L}^{2}(\Omega)\right) \wedge \mathrm{w}-\mathrm{L}_{\text {loc }}^{2}\left([0, \infty) ; \mathrm{w}-\mathrm{H}^{1}(\Omega)\right) .
$$

Remark 5 We denote by " $\wedge$ " the intersection of topological spaces equipped with the relative topology induced by the inclusion maps. The spaces here are first countable topological vector spaces which implies that a sequence converges in the intersection if and only if it converges in each space separately, and furthermore that a set is relatively compact in the intersection if and only if it is relatively compact in each space separately. The sense of convergence in the particular spaces we are using here can be summarized as follows. Given a topological vector space $\mathrm{X}$ with dual $\mathrm{X}^{\prime}$, a sequence of functions $\varphi_{n}:[0, \infty) \rightarrow \mathrm{X}$ converges to $\varphi$ in $\mathrm{C}([0, \infty)$; w-X), where $\mathrm{w}$-X denotes $\mathrm{X}$ endowed with its weak topology, when for every $\psi \in \mathrm{X}^{\prime}$

$$
\left\langle\psi, \varphi_{n}(t)\right\rangle_{\mathrm{X}^{\prime}, \mathrm{X}} \rightarrow\langle\psi, \varphi(t)\rangle_{\mathrm{X}^{\prime}, \mathrm{X}}
$$

uniformly on compact subsets of $[0, \infty)$. Similarly, $\varphi_{n} \rightarrow \varphi$ in w- $\mathrm{L}_{\text {loc }}^{p}([0, \infty) ; \mathrm{w}-\mathrm{X})$ when for every $T>0$ and $\psi \in \mathrm{L}_{\mathrm{loc}}^{q}\left([0, \infty) ; \mathrm{X}^{\prime}\right)$

$$
\int_{0}^{T}\left\langle\psi(t), \varphi_{n}(t)\right\rangle_{\mathrm{X}^{\prime}, \mathrm{X}} \mathrm{d} t \rightarrow \int_{0}^{T}\langle\psi(t), \varphi(t)\rangle_{\mathrm{X}^{\prime}, \mathrm{X}} \mathrm{d} t,
$$

where $q=p /(p-1)$ is the Hölder conjugate of $p$, and $p \in[1, \infty)$.

Proof of Step 2. We first derive a $\mathrm{L}^{2}$ estimate for the regularized equation (4.12a) by taking its scalar product with $\omega_{n}$, integrating the dissipative term by parts and observing that the nonlinear term vanishes according to (4.4). Hence,

$$
\frac{\mathrm{d}}{\mathrm{d} t}\left\|\omega_{n}\right\|_{\mathrm{L}^{2}}^{2}=-2 \nu\left\|\nabla \omega_{n}\right\|_{\mathrm{L}^{2}}^{2} .
$$

Integration in time gives

$$
\left\|\omega_{n}(t)\right\|_{\mathrm{L}^{2}}^{2}=\left\|\omega_{n}^{\mathrm{in}}\right\|_{\mathrm{L}^{2}}^{2}-2 \nu \int_{0}^{t}\left\|\nabla \omega_{n}\left(t^{\prime}\right)\right\|_{\mathrm{L}^{2}}^{2} \mathrm{~d} t^{\prime},
$$

which implies the estimates

$$
\left\|\omega_{n}(t)\right\|_{\mathrm{L}^{2}} \leq\left\|\omega_{n}^{\mathrm{in}}\right\|_{\mathrm{L}^{2}} \leq\left\|\omega^{\mathrm{in}}\right\|_{\mathrm{L}^{2}},
$$


and

$$
\int_{0}^{t}\left\|\nabla \omega_{n}\left(t^{\prime}\right)\right\|_{\mathrm{L}^{2}}^{2} \mathrm{~d} t^{\prime} \leq \frac{1}{2 \nu}\left\|\omega^{\mathrm{in}}\right\|_{\mathrm{L}^{2}}^{2}
$$

This shows that the sequence $\left\{\omega_{n}\right\}$ is a norm bounded set in $\mathrm{L}_{\text {loc }}^{2}\left([0, \infty) ; \mathrm{H}_{0}^{1}(\Omega)\right)$ and thus relatively compact in $\mathrm{w}-\mathrm{L}_{\text {loc }}^{2}\left([0, \infty) ; \mathrm{w}-\mathrm{H}_{0}^{1}(\Omega)\right)$, because norm bounded sets are relatively compact in the weak-* topology which is identical to the weak topology on a reflexive space.

It remains to be shown that $\left\{\omega_{n}\right\}$ is relatively compact in $\mathrm{C}\left([0, \infty)\right.$; w- $\left.\mathrm{L}^{2}(\Omega)\right)$. According to the Arzela-Ascoli theorem this is equivalent to the following.

(i) $\left\{\omega_{n}(t)\right\}$ is a relatively compact set in $\mathrm{w}-\mathrm{L}^{2}(\Omega)$ for every $t \geq 0$;

(ii) $\left\{\omega_{n}\right\}$ is equicontinuous in $\mathrm{C}\left([0, \infty)\right.$; w- $\left.\mathrm{L}^{2}(\Omega)\right)$, i.e. for every $\psi \in \mathrm{L}^{2}(\Omega)$ the sequence $\left\{\left\langle\psi \omega_{n}\right\rangle\right\}$ is equicontinuous in $\mathrm{C}([0, \infty))$.

Condition (i) is equivalent to boundedness in $\mathrm{L}^{2}(\Omega)$ for every $t \geq 0$, which follows from estimate (4.20).

In order to show (ii), we begin by considering the case where $\psi$ is a finite linear combination of eigenvectors of $A$. One obtains

$$
\begin{aligned}
& \left|\left\langle\psi \omega_{n}\left(t_{2}\right)\right\rangle-\left\langle\psi \omega_{n}\left(t_{1}\right)\right\rangle\right| \\
= & \left|\nu \int_{t_{1}}^{t_{2}}\left\langle\boldsymbol{\nabla} \psi \cdot \nabla \omega_{n}\right\rangle \mathrm{d} t-\int_{t_{1}}^{t_{2}}\left\langle\left(\boldsymbol{u}_{n} \cdot \nabla \psi_{n}\right) \omega_{n}\right\rangle \mathrm{d} t\right| \\
\leq & \nu\|\nabla \psi\|_{\mathrm{L}^{\infty}}\left(\int_{t_{1}}^{t_{2}} \mathrm{~d} t\right)^{1 / 2}\left(\int_{t_{1}}^{t_{2}}\left\|\omega_{n}\right\|_{\mathrm{H}^{1}}^{2} \mathrm{~d} t\right)^{1 / 2} \\
& +\left\|\nabla \psi_{n}\right\|_{\mathrm{L}^{\infty}}\left(\int_{t_{1}}^{t_{2}} \mathrm{~d} t\right)^{1 / 4}\left(\int_{t_{1}}^{t_{2}}\left\|\boldsymbol{u}_{n}\right\|_{\mathrm{L}^{4}}^{4} \mathrm{~d} t\right)^{1 / 4}\left(\int_{t_{1}}^{t_{2}}\left\|\omega_{n}\right\|_{\mathrm{L}^{2}}^{2} \mathrm{~d} t\right)^{1 / 2},
\end{aligned}
$$

where $\psi_{n} \equiv P_{n} \psi=\psi$ for $n$ large enough. The sequence $\left\{\omega_{n}\right\}$ is bounded in $\mathrm{L}_{\text {loc }}^{\infty}\left([0, \infty) ; \mathrm{L}^{2}(\Omega)\right)$, hence $\left\{\boldsymbol{u}_{n}\right\}$ is bounded in $\mathrm{L}_{\text {loc }}^{4}\left([0, \infty) ; \mathrm{L}^{4}\left(\Omega, \mathbb{R}^{2}\right)\right)$. Furthermore, $\left\{\omega_{n}\right\}$ is bounded in $\mathrm{L}_{\text {loc }}^{2}\left([0, \infty) ; \mathrm{H}_{0}^{1}(\Omega)\right)$, so that for every $n \in \mathbb{N}, T<\infty$ and $\left[t_{1}, t_{2}\right] \subset[0, T]$,

$$
\begin{aligned}
\left|\left\langle\psi \omega_{n}\left(t_{2}\right)\right\rangle-\left\langle\psi \omega_{n}\left(t_{1}\right)\right\rangle\right| \leq c\left(\psi, \nu, T, \omega^{\text {in }}\right)\left|t_{2}-t_{1}\right|^{1 / 2} & \\
& +c\left(\psi, T, \omega^{\text {in }}\right)\left|t_{2}-t_{1}\right|^{1 / 4} .
\end{aligned}
$$

Because the right side of this equation is independent of $n$, the set $\left\{\left\langle\psi \omega_{n}\right\rangle\right\}$ is equicontinuous in $\mathrm{C}([0, \infty))$.

We extend the class of test functions to $\mathrm{L}^{2}(\Omega)$ with a density argument. Let $\varepsilon>0$. Choose $\psi_{\varepsilon}$ to be a finite linear combination of eigenvectors of $A$ such that

$$
\left\|\psi-\psi_{\varepsilon}\right\|_{\mathrm{L}^{2}}<\frac{\varepsilon}{3\left\|\omega^{\mathrm{in}}\right\|_{\mathrm{L}^{2}}+1} .
$$


By the triangle inequality

$$
\begin{aligned}
& \left|\left\langle\psi \omega_{n}\left(t_{2}\right)\right\rangle-\left\langle\psi \omega_{n}\left(t_{1}\right)\right\rangle\right| \\
= & \left|\left\langle\left(\psi-\psi_{\varepsilon}\right)\left(\omega_{n}\left(t_{2}\right)-\omega_{n}\left(t_{1}\right)\right)\right\rangle+\left\langle\psi_{\varepsilon}\left(\omega_{n}\left(t_{2}\right)-\omega_{n}\left(t_{1}\right)\right)\right\rangle\right| \\
\leq & \left\|\psi-\psi_{\varepsilon}\right\|_{L^{2}}\left(\left\|\omega_{n}\left(t_{2}\right)\right\|_{L^{2}}+\left\|\omega_{n}\left(t_{1}\right)\right\|_{L^{2}}\right)+\left|\left\langle\psi_{\varepsilon}\left(\omega_{n}\left(t_{2}\right)-\omega_{n}\left(t_{1}\right)\right)\right\rangle\right| .
\end{aligned}
$$

From (4.24) and the uniform $\mathrm{L}^{2}$ bound (4.20) we infer that the first term on the right is smaller that $\frac{2}{3} \varepsilon$. Applying (4.23) and choosing $\left|t_{2}-t_{1}\right|$ small enough, the second term on the right can be made smaller than $\frac{1}{3} \varepsilon$, so that the whole expression is less than $\varepsilon$. This establishes (ii) and completes the proof of Step 2.

Step 3 Show that $\left\{\omega_{n}\right\}$ is a relatively compact set in $\mathrm{L}_{\text {loc }}^{2}\left([0, \infty) ; \mathrm{L}^{2}(\Omega)\right)$ endowed with its strong topology.

Proof of Step 3. The statement follows directly from the fact that the embedding

$$
\mathrm{C}\left([0, \infty) ; \mathrm{w}-\mathrm{L}^{2}(\Omega)\right) \wedge \mathrm{w}-\mathrm{L}_{\text {loc }}^{2}\left([0, \infty) ; \mathrm{w}-\mathrm{H}^{1}(\Omega)\right) \hookrightarrow \mathrm{L}_{\text {loc }}^{2}\left([0, \infty) ; \mathrm{L}^{2}(\Omega)\right)
$$

is continuous. This can be established as in [8].

Step 4 Show that there is a subsequence of $\left\{\omega_{n}\right\}$ converging to a function $\omega$ which satisfies the weak form (4.8) and the energy relation (4.13).

Proof of Step 4. Steps 2 and 3 assert that there exists a subsequence of $\left\{\omega_{n}\right\}$ which we will still denote by $\left\{\omega_{n}\right\}$ that converges to a function

$$
\omega \in \mathrm{C}\left([0, \infty) ; \mathrm{w}-\mathrm{L}^{2}(\Omega)\right) \cap \mathrm{L}_{\text {loc }}^{2}\left([0, \infty) ; \mathrm{H}_{0}^{1}(\Omega)\right)
$$

in the following sense:

(i) $\omega_{n} \rightarrow \omega$ in $\mathrm{C}\left([0, \infty) ; \mathrm{w}-\mathrm{L}^{2}(\Omega)\right)$;

(ii) $\omega_{n} \rightarrow \omega$ in $\mathrm{w}-\mathrm{L}_{\text {loc }}^{2}\left([0, \infty) ; \mathrm{w}-\mathrm{H}_{0}^{1}(\Omega)\right)$;

(iii) $\omega_{n} \rightarrow \omega$ in $\mathrm{L}_{\text {loc }}^{2}\left([0, \infty) ; \mathrm{L}^{2}(\Omega)\right)$.

Note that $\omega \in \mathrm{L}_{\text {loc }}^{2}\left([0, \infty) ; \mathrm{H}_{0}^{1}(\Omega)\right)$ as opposed to $\mathrm{L}_{\text {loc }}^{2}\left([0, \infty) ; \mathrm{H}^{1}(\Omega)\right)$ because $\mathrm{H}_{0}^{1}(\Omega)$ is a closed subspace of $\mathrm{H}^{1}(\Omega)$ and $\omega_{n}(t) \in \mathrm{H}_{0}^{1}(\Omega)$ for every $n$ and $t$.

It remains to be shown that $\omega$ satisfies the weak form (4.8a). Towards this end, we show that for every test function $\psi$ from a dense subset of $H_{0}^{1}$ each of 
the terms in the regularized weak form (4.14) converges to the corresponding term in (4.8a). This shows that $\omega$ satisfies the weak form for any such $\psi$ and, by density, for every $\psi \in \mathrm{H}_{0}^{1}(\Omega)$.

This said, it suffices to assume a test function $\psi$ which is a finite linear combination of eigenvectors of $A$. The convergence (i) in $\mathrm{C}\left([0, \infty) ; \mathrm{w}-\mathrm{L}^{2}(\Omega)\right)$ implies that

$$
\left\langle\psi \omega_{n}(t)\right\rangle \rightarrow\langle\psi \omega(t)\rangle
$$

for every $t \geq 0$ as $n \rightarrow \infty$. Convergence (ii) in $\mathrm{w}_{-} \mathrm{L}_{\text {loc }}^{2}\left([0, \infty) ; \mathrm{w}-\mathrm{H}_{0}^{1}(\Omega)\right)$ yields

$$
\int_{t_{1}}^{t_{2}}\left\langle\nabla \psi \cdot \nabla \omega_{n}\right\rangle \mathrm{d} t \rightarrow \int_{t_{1}}^{t_{2}}\langle\nabla \psi \cdot \nabla \omega\rangle \mathrm{d} t .
$$

To show the convergence of the nonlinear term we use the estimate

$$
\begin{aligned}
& \int_{t_{1}}^{t_{2}}\left(\left\langle\left(\boldsymbol{u}_{n} \cdot \boldsymbol{\nabla} \psi_{n}\right) \omega_{n}\right\rangle-\langle(\boldsymbol{u} \cdot \boldsymbol{\nabla} \psi) \omega\rangle\right) \mathrm{d} t \\
& =\int_{t_{1}}^{t_{2}}\left(\left\langle\left(\left(\boldsymbol{u}_{n}-\boldsymbol{u}\right) \cdot \nabla \psi_{n}\right) \omega_{n}\right\rangle+\left\langle\left(\boldsymbol{u} \cdot \boldsymbol{\nabla}\left(\psi_{n}-\psi\right)\right) \omega_{n}\right\rangle+\left\langle(\boldsymbol{u} \cdot \nabla \psi)\left(\omega_{n}-\omega\right)\right\rangle\right) \mathrm{d} t \\
& \leq \int_{t_{1}}^{t_{2}}\left(\left\|\boldsymbol{u}_{n}-\boldsymbol{u}\right\|_{\mathrm{L}^{2}}\left\|\nabla \psi_{n}\right\|_{\mathrm{L}^{\infty}}\left\|\omega_{n}\right\|_{\mathrm{L}^{2}}+\|\boldsymbol{u}\|_{\mathrm{L}^{2}}\left\|\nabla\left(\psi_{n}-\psi\right)\right\|_{\mathrm{L}^{\infty}}\left\|\omega_{n}\right\|_{\mathrm{L}^{2}}\right) \mathrm{d} t \\
& \quad+\left|\int_{t_{1}}^{t_{2}}\left\langle(\boldsymbol{u} \cdot \nabla \psi)\left(\omega_{n}-\omega\right)\right\rangle \mathrm{d} t\right|
\end{aligned}
$$

Thus, in order to pass to the limit it is sufficient to have strong convergence of $\left\{\boldsymbol{u}_{n}\right\}$ in $\mathrm{L}_{\text {loc }}^{2}\left([0, \infty) ; \mathrm{L}^{2}\left(\Omega, \mathbb{R}^{2}\right)\right)$ and weak convergence of $\left\{\omega_{n}\right\}$ in $\mathrm{L}_{\text {loc }}^{2}\left([0, \infty) ; \mathrm{L}^{2}(\Omega)\right)$, properties that follow directly from (ii) and (iii).

Finally, the convergence of the energy relation (4.8) follows from the fact that $\omega_{n}^{\text {in }} \rightarrow \omega^{\text {in }}$ in $\mathrm{L}^{2}$ and that the convergence (i) implies

$$
\|\omega(t)\|_{\mathrm{L}^{2}} \leq \liminf _{n \rightarrow \infty}\left\|\omega_{n}(t)\right\|_{\mathrm{L}^{2}},
$$

for every $t \geq 0$. This completes the proof of Step 4 .

The first four steps constitute the minimal construction necessary to obtain an existence, but not necessarily a uniqueness result via an equicontinuity argument. These steps do not depend on any particular spatial dimension. However, uniqueness can be shown in the two dimensional case. For passing to the limit $\nu \rightarrow 0$ in Theorem 7 below, we also need some further regularity, the "moreover" part in the statement of the theorem. 
Step 5 Show that for initial values $\omega \in \mathrm{H}_{0}^{1}(\Omega)$, the sequence $\left\{\omega_{n}\right\}$ is a relatively compact set in

$$
\mathrm{C}\left([0, \infty) ; \mathrm{w}-\mathrm{H}_{0}^{1}(\Omega)\right) \wedge \mathrm{w}-\mathrm{L}_{\text {loc }}^{2}\left([0, \infty) ; \mathrm{w}-\mathrm{H}^{2}(\Omega)\right) .
$$

Proof of Step 5. We take the $\mathrm{L}^{2}$ scalar product of the regularized equation (4.12a) with $A \omega_{n}$ and integrate by parts to obtain

$$
\frac{1}{2} \frac{\mathrm{d}}{\mathrm{d} t}\left\|\nabla \omega_{n}\right\|_{\mathrm{L}^{2}}^{2}+\nu\left\|A \omega_{n}\right\|_{\mathrm{L}^{2}}^{2}=\left\langle\left(\boldsymbol{u}_{n} \cdot \nabla \omega_{n}\right) A \omega_{n}\right\rangle .
$$

The nonlinear term is split up with a Hölder and a Young inequality. Moreover, in two dimensions the $\mathrm{L}^{\infty}$ norm is controlled by the $\mathrm{H}^{2}$ norm. Invoking the continuity properties of $K$ we get

$$
\begin{aligned}
\left|\left\langle\left(\boldsymbol{u}_{n} \cdot \nabla \omega_{n}\right) A \omega_{n}\right\rangle\right| & \leq \frac{2}{\nu}\left\|\boldsymbol{u}_{n}\right\|_{\mathrm{L}^{\infty}}^{2}\left\|\nabla \omega_{n}\right\|_{\mathrm{L}^{2}}^{2}+\frac{\nu}{2}\left\|A \omega_{n}\right\|_{\mathrm{L}^{2}}^{2} \\
& \leq \frac{2 c}{\nu}\left\|\omega_{n}\right\|_{\mathrm{H}^{1}}^{2}\left\|\nabla \omega_{n}\right\|_{\mathrm{L}^{2}}^{2}+\frac{\nu}{2}\left\|A \omega_{n}\right\|_{\mathrm{L}^{2}}^{2},
\end{aligned}
$$

hence

$$
\frac{\mathrm{d}}{\mathrm{d} t}\left\|\nabla \omega_{n}\right\|_{\mathrm{L}^{2}}^{2}+\nu\left\|A \omega_{n}\right\|_{\mathrm{L}^{2}}^{2} \leq \frac{4 c}{\nu}\left\|\omega_{n}\right\|_{\mathrm{H}^{1}}^{2}\left\|\nabla \omega_{n}\right\|_{\mathrm{L}^{2}}^{2} .
$$

After neglecting the second term on the left, this differential inequality can easily be solved, resulting in the bound

$$
\left\|\nabla \omega_{n}(t)\right\|_{\mathrm{L}^{2}}^{2} \leq\left\|\nabla \omega_{n}^{\mathrm{in}}\right\|_{\mathrm{L}^{2}}^{2} \exp \left(\frac{4 c}{\nu} \int_{0}^{t}\left\|\omega_{n}\left(t^{\prime}\right)\right\|_{\mathrm{H}^{1}}^{2} \mathrm{~d} t^{\prime}\right) .
$$

We recall that $(A \cdot, \cdot)_{\mathrm{L}^{2}}$ defines a scalar product on $\mathrm{H}_{0}^{1}(\Omega)$ with respect to which the projectors $P_{n}$ are clearly orthogonal. This means that $\left\|\nabla \omega_{n}^{\text {in }}\right\|_{\mathrm{L}^{2}} \leq\left\|\nabla \omega^{\text {in }}\right\|_{\mathrm{L}^{2}}$. It was already shown in Step 2 that $\left\{\omega_{n}\right\}$ is a bounded set in $\mathrm{L}_{\text {loc }}^{2}\left([0, \infty) ; \mathrm{H}_{0}^{1}(\Omega)\right)$. Therefore estimate (4.35) shows that $\left\{\omega_{n}\right\}$ is a bounded set in $\mathrm{L}_{\text {loc }}^{\infty}\left([0, \infty) ; \mathrm{H}_{0}^{1}(\Omega)\right)$. In turn, we see that the right side of (4.34) is bounded, so that a second estimate can be obtained by integrating inequality (4.34) in time:

$$
\left\|\nabla \omega_{n}(t)\right\|_{\mathrm{L}^{2}}^{2}-\left\|\nabla \omega_{n}^{\mathrm{in}}\right\|_{\mathrm{L}^{2}}^{2}+\nu \int_{0}^{t}\left\|A \omega_{n}\left(t^{\prime}\right)\right\|_{\mathrm{L}^{2}}^{2} \mathrm{~d} t^{\prime} \leq \frac{4 c t}{\nu} \sup _{t^{\prime} \in[0, t]}\left\|\omega_{n}\left(t^{\prime}\right)\right\|_{\mathrm{H}^{1}}^{4} .
$$

I.e., the sequence $\left\{\omega_{n}\right\}$ is a bounded set in $\mathrm{L}_{\text {loc }}^{2}\left([0, \infty) ; \mathrm{H}^{2}(\Omega)\right)$ and, hence, is relatively compact in its weak-* topology, which on this reflexive space is the same as the weak topology.

Relative compactness in $\mathrm{C}\left([0, \infty) ; \mathrm{w}-\mathrm{H}_{0}^{1}(\Omega)\right)$ is proved by an equicontinuity argument as in Step 2. The pointwise boundedness is now guaranteed by 
the bound (4.35) while the weak equicontinuity is proved exactly as before, using the $\mathrm{H}^{1}$ scalar product and a test function $\psi \in \mathrm{H}^{-1}(\Omega)$ in the density argument.

Step 6 Show that $\left\{\omega_{n}\right\}$ is a relatively compact set in $\mathrm{L}_{\text {loc }}^{2}\left([0, \infty) ; \mathrm{H}_{0}^{1}(\Omega)\right)$.

This statement is directly analogous to Step 3 and is proved the same way.

Step 7 Pass to the limit. That is, identify the solution $\omega$ obtained in Step 4 with the limit of a convergent subsequence of $\left\{\omega_{n}\right\}$ in a set of stronger spaces, and use this information to recover the energy relation (4.10) from a regularized $\mathrm{L}^{p}$ estimate.

Proof of Step 7. Steps 5 and 6 assert that there exists a subsequence of $\left\{\omega_{n}\right\}$ which we will still denote by $\left\{\omega_{n}\right\}$ that converges to a function $\omega$, that is now contained in

$$
\omega \in \mathrm{C}\left([0, \infty) ; \mathrm{w}-\mathrm{H}_{0}^{1}(\Omega)\right) \cap \mathrm{L}_{\text {loc }}^{2}\left([0, \infty) ; \mathrm{H}^{2}(\Omega) \cap \mathrm{H}_{0}^{1}(\Omega)\right),
$$

in the following sense:

(i) $\omega_{n} \rightarrow \omega$ in $\mathrm{C}\left([0, \infty)\right.$; $\left.\mathrm{w}-\mathrm{H}_{0}^{1}(\Omega)\right)$;

(ii) $\omega_{n} \rightarrow \omega$ in $\mathrm{w}_{-} \mathrm{L}_{\text {loc }}^{2}\left([0, \infty) ; \mathrm{w}-\mathrm{H}^{2}(\Omega)\right)$;

(iii) $\omega_{n} \rightarrow \omega$ in $\mathrm{L}_{\text {loc }}^{2}\left([0, \infty) ; \mathrm{H}_{0}^{1}(\Omega)\right)$.

According to Step 4, $\omega$ satisfies the weak form (4.8a). In order to prove that $\omega$ also satisfies the energy relation (4.10), we first derive a $L^{p}$ estimate for the regularized system and then show that we can obtain (4.10) in the limit when $n \rightarrow \infty$.

Let $p \geq 2$ be an even integer, take the scalar product of the regularized form (4.12a) with $\omega_{n}^{p-1}$ and integrate by parts to obtain

$$
\begin{aligned}
& \frac{1}{p} \frac{\mathrm{d}}{\mathrm{d} t}\left\|\omega_{n}\right\|_{\mathrm{L}^{p}}^{p}+\nu(p-1)\left\langle\left(\nabla \omega_{n} \cdot \nabla \omega_{n}\right) \omega_{n}^{p-2}\right\rangle+\left\langle\left(\boldsymbol{u}_{n} \cdot \nabla \omega_{n}\right) P_{n} \omega_{n}^{p-1}\right\rangle \\
= & 0 .
\end{aligned}
$$

The second term is always positive and, hence, can be neglected. Then integration in time gives

$$
\left\|\omega_{n}(t)\right\|_{L^{p}}^{p} \leq\left\|\omega_{n}^{\text {in }}\right\|_{L^{p}}^{p}-p \int_{0}^{t}\left\langle\left(\boldsymbol{u}_{n} \cdot \nabla \omega_{n}\right) P_{n} \omega_{n}^{p-1}\right\rangle \mathrm{d} t^{\prime} .
$$

We claim that this relation converges to (4.10) as $n \rightarrow \infty$. First, the convergence in $\mathrm{C}\left([0, \infty) ; \mathrm{w}-\mathrm{H}^{1}(\Omega)\right)$ implies convergence in $\mathrm{C}\left([0, \infty) ; \mathrm{w}^{-} \mathrm{L}^{p}(\Omega)\right)$ and therefore

$$
\|\omega(t)\|_{\mathrm{L}^{p}} \leq \liminf _{n \rightarrow \infty}\left\|\omega_{n}(t)\right\|_{\mathrm{L}^{p}}
$$


Second, $\omega_{n}^{\text {in }}$ converges to $\omega^{\text {in }}$ in $\mathrm{L}^{p}$ because

$$
\left\|\omega_{n}^{\text {in }}-\omega^{\text {in }}\right\|_{L^{p}} \leq c\left\|\omega_{n}^{\text {in }}-\omega^{\text {in }}\right\|_{\mathrm{H}^{1}},
$$

and the $P_{n}$ are also orthogonal in $H_{0}^{1}$, so that the right side converges.

Lastly, we estimate by using a Hölder inequality, Sobolev embedding theorems and the continuity properties of $K$ that

$$
\begin{aligned}
\left|\left\langle\left(\boldsymbol{u}_{n} \cdot \nabla \omega_{n}\right) P_{n} \omega_{n}^{p-1}\right\rangle\right| & \leq\left\|\boldsymbol{u}_{n}\right\|_{L^{\infty}}\left\|\nabla \omega_{n}\right\|_{L^{2}}\left\|P_{n}\right\|_{\mathcal{L}\left(\mathrm{L}^{2}\right)}\left\|\omega_{n}^{p-1}\right\|_{L^{2}} \\
& \leq c\left\|\omega_{n}\right\|_{\mathrm{H}^{1}}^{p+1}\left\|P_{n}\right\|_{\mathcal{L}\left(\mathrm{L}^{2}\right)} .
\end{aligned}
$$

Thus, the term inside the absolute value signs can be viewed as a continuous multilinear form on $\left(\mathrm{H}^{1}\right)^{p+1} \times \mathcal{L}\left(\mathrm{L}^{\epsilon}\right)$. Because of this the strong convergence $\omega_{n} \rightarrow \omega$ in $\mathrm{L}_{\mathrm{loc}}^{\infty}\left([0, \infty) ; \mathrm{H}_{0}^{1}(\Omega)\right)$ combined with the pointwise convergence $P_{n} \rightarrow I$ in $\mathrm{L}^{2}(\Omega)$ imply that

$$
\int_{0}^{t}\left\langle\left(\boldsymbol{u}_{n} \cdot \nabla \omega_{n}\right) P_{n} \omega_{n}^{p-1}\right\rangle \mathrm{d} t^{\prime} \rightarrow \int_{0}^{t}\left\langle(\boldsymbol{u} \cdot \nabla \omega) \omega^{p-1}\right\rangle \mathrm{d} t^{\prime} .
$$

Integration by parts analogous to the steps leading up to equation (4.4) immediately shows that the right side is zero. This concludes the proof of Step 7 .

Step 8 Show that $\omega \in \mathrm{C}\left([0, \infty) ; \mathrm{H}_{0}^{1}(\Omega)\right)$.

Proof of Step 8. Step 8 follows from the facts that the weak form (4.8a) implies the existence of a distributional time derivative $\partial_{t} \omega \in \mathrm{L}_{\mathrm{loc}}^{2}\left([0, \infty) ; \mathrm{L}^{2}(\Omega)\right)$ and that the embedding

$$
\begin{gathered}
\left\{\varphi \in \mathrm{L}_{\text {loc }}^{2}\left([0, \infty) ; \mathrm{H}^{2}(\Omega) \cap \mathrm{H}_{0}^{1}(\Omega)\right): \partial_{t} \varphi \in \mathrm{L}_{\text {loc }}^{2}\left([0, \infty) ; \mathrm{L}^{2}(\Omega)\right)\right\} \\
\hookrightarrow \mathrm{C}\left([0, \infty) ; \mathrm{H}_{0}^{1}(\Omega)\right)
\end{gathered}
$$

is continuous. The latter statement easily follows from more general results as proved in [21, Theorem 25.5]. For the former we need to recover the time derivative of $\omega$ in a weak sense. From the estimates

$$
\int_{0}^{t}\left|\left\langle\nabla \psi \cdot \nabla \omega\left(t^{\prime}\right)\right\rangle\right|^{2} \mathrm{~d} t^{\prime} \leq\|\psi\|_{\mathrm{L}^{2}}^{2} \int_{0}^{t}\left\|A \omega\left(t^{\prime}\right)\right\|_{\mathrm{L}^{2}}^{2} \mathrm{~d} t^{\prime}
$$

and

$$
\int_{0}^{t}\left|\left\langle\left(\boldsymbol{u}\left(t^{\prime}\right) \cdot \nabla \psi\right) \omega\left(t^{\prime}\right)\right\rangle\right|^{2} \mathrm{~d} t^{\prime} \leq\|\psi\|_{\mathrm{L}^{2}}^{2} \int_{0}^{t}\left\|\boldsymbol{u}\left(t^{\prime}\right)\right\|_{\mathrm{L}^{4}}^{2}\left\|\nabla \omega\left(t^{\prime}\right)\right\|_{\mathrm{L}^{4}}^{2} \mathrm{~d} t^{\prime}
$$


noting that the time integrals on the respective right sides are finite, we conclude that the mapping

$$
(t, \psi) \mapsto \nu\langle\nabla \psi \cdot \nabla \omega\rangle-\langle(\boldsymbol{u}(t) \cdot \nabla \psi) \omega(t)\rangle
$$

defines a function in $\mathrm{L}_{\text {loc }}^{2}\left([0, \infty) ; \mathrm{L}^{2}(\Omega)\right)$. Let $\psi \in \mathrm{L}^{2}(\Omega)$. The weak form (4.8a) includes in particular

$$
\langle\psi \omega(t)\rangle=\left\langle\psi \omega^{\text {in }}\right\rangle-\nu \int_{0}^{t}\langle\nabla \psi \cdot \nabla \omega\rangle \mathrm{d} t^{\prime}+\int_{0}^{t}\langle(\boldsymbol{u} \cdot \nabla \psi) \omega\rangle \mathrm{d} t^{\prime} .
$$

Take a test function $\theta \in C_{0}^{\infty}([0, \infty))$, multiply the previous line with $\mathrm{d} \theta / \mathrm{d} t$, integrate from 0 to $\infty$ and then integrate by parts to obtain

$$
\begin{aligned}
\int_{0}^{\infty}\langle\psi \omega(t)\rangle \frac{\mathrm{d} \theta}{\mathrm{d} t} \mathrm{~d} t=- & \nu \int_{0}^{\infty}\langle\boldsymbol{\nabla} \psi \cdot \nabla \omega(t)\rangle \theta(t) \mathrm{d} t \\
& +\int_{0}^{\infty}\langle(\boldsymbol{u}(t) \cdot \boldsymbol{\nabla} \psi) \omega(t)\rangle \theta(t) \mathrm{d} t .
\end{aligned}
$$

Integration by parts is legitimate here because from our earlier remark it follows that the integrand on the right side of $(4.48)$ is a $L_{\mathrm{loc}}^{1}([0, \infty))$ function. Relation (4.49) is equivalent to saying that

$$
\frac{\mathrm{d}}{\mathrm{d} t}\langle\psi \omega(t)\rangle=-\nu\langle\nabla \psi \cdot \nabla \omega(t)\rangle+\langle(\boldsymbol{u}(t) \cdot \nabla \psi) \omega(t)\rangle
$$

in the sense of distributions on $[0, \infty)$, so that we can write

$$
\partial_{t} \omega=-\nu A \omega-\boldsymbol{u} \cdot \nabla \omega \in \mathrm{L}_{\mathrm{loc}}^{2}\left([0, \infty) ; \mathrm{L}^{2}(\Omega)\right) .
$$

This completes the proof of Step 8.

Step 9 Show that the solution $\omega$ is unique.

Proof of Step 9. Assume that $\hat{\omega}$ and $\bar{\omega}$ are two solutions to equation (4.8) and set $\hat{\boldsymbol{u}}=K \hat{\omega}, \overline{\boldsymbol{u}}=K \bar{\omega}$ and $\varphi=\hat{\omega}-\bar{\omega}$. From (4.51) we infer that

$$
\begin{aligned}
& \frac{1}{2} \frac{\mathrm{d}}{\mathrm{d} t}\|\varphi(t)\|_{\mathrm{L}^{2}}^{2}+\nu\langle A \varphi, \varphi\rangle \\
= & -\langle(\hat{\boldsymbol{u}} \cdot \boldsymbol{\nabla} \hat{\omega}) \varphi\rangle+\langle(\overline{\boldsymbol{u}} \cdot \boldsymbol{\nabla} \bar{\omega}) \varphi\rangle \\
= & -\langle((\hat{\boldsymbol{u}}-\overline{\boldsymbol{u}}) \cdot \boldsymbol{\nabla} \hat{\omega}) \varphi\rangle-\langle(\overline{\boldsymbol{u}} \cdot \boldsymbol{\nabla} \varphi) \varphi\rangle .
\end{aligned}
$$


The second term on the right vanishes due to (4.4), so that one has the estimate

$$
\begin{aligned}
& \frac{1}{2} \frac{\mathrm{d}}{\mathrm{d} t}\|\varphi(t)\|_{\mathrm{L}^{2}}^{2}+\nu\|\varphi(t)\|_{\mathrm{H}^{1}}^{2} \\
\leq & \|\hat{\boldsymbol{u}}-\overline{\boldsymbol{u}}\|_{\mathrm{L}^{4}}\|\boldsymbol{\nabla} \hat{\omega}\|_{\mathrm{L}^{2}}\|\varphi\|_{\mathrm{L}^{4}} \\
\leq & c_{1}\|\hat{\boldsymbol{u}}-\overline{\boldsymbol{u}}\|_{\mathrm{L}^{2}}^{1 / 2}\|\hat{\boldsymbol{u}}-\overline{\boldsymbol{u}}\|_{\mathrm{H}^{1}}^{1 / 2}\|\hat{\omega}\|_{\mathrm{H}^{1}}\|\varphi\|_{\mathrm{L}^{2}}^{1 / 2}\|\varphi\|_{\mathrm{H}^{1}}^{1 / 2} \\
\leq & c_{2}\|\varphi\|_{\mathrm{L}^{2}}^{3 / 2}\|\hat{\omega}\|_{\mathrm{H}^{1}}\|\varphi\|_{\mathrm{H}^{1}}^{1 / 2} .
\end{aligned}
$$

The second inequality has been achieved by a Sobolev embedding and the third inequality holds due to the continuity of $K$. Now, applying the Young inequality such that $\nu\|\varphi(t)\|_{\mathrm{H}^{1}}$ terms cancels from both sides one obtains

$$
\frac{\mathrm{d}}{\mathrm{d} t}\|\varphi(t)\|_{\mathrm{L}^{2}}^{2} \leq c_{3}(\nu)\|\varphi(t)\|_{\mathrm{L}^{2}}^{2}\|\hat{\omega}(t)\|_{\mathrm{H}^{1}}^{4 / 3} .
$$

This linear differential inequality can be integrated directly and yields

$$
\|\varphi(t)\|_{\mathrm{L}^{2}}^{2} \leq\|\varphi(0)\|_{\mathrm{L}^{2}}^{2} \exp \left(c_{3}(\nu) \int_{0}^{t}\left\|\hat{\omega}\left(t^{\prime}\right)\right\|_{\mathrm{H}^{1}}^{4 / 3} \mathrm{~d} t^{\prime}\right) .
$$

Since $\hat{\omega} \in \mathrm{L}_{\text {loc }}^{2}\left([0, \infty) ; \mathrm{H}^{1}(\Omega)\right)$, the exponential factor is finite, and hence the difference $\varphi$ between the two solutions remains zero if it is so initially.

Remark 6 Uniqueness still holds when $\omega^{\text {in }} \in \mathrm{L}^{2}(\Omega)$. It is easy to check that in this case $(4.51)$ weakens to $\partial_{t} \omega \in \mathrm{L}_{\text {loc }}^{2}\left([0, \infty) ; \mathrm{H}^{-1}(\Omega)\right)$, so that $\langle\cdot\rangle$ in (4.52) has to be interpreted as the duality product between $\mathrm{H}_{0}^{1}$ and $\mathrm{H}^{-1}$, and (4.53) has to be argued by density. The rest of the proof can remain unchanged.

5. The inviscid limit. In this section we will show that it is possible to pass to the limit $\nu \rightarrow 0$ in Theorem 6 . We obtain the following theorem on the inviscid equations.

Theorem 7 Given $\omega^{\text {in }} \in \mathrm{L}^{2}(\Omega)$, there exists a function

$$
\omega \in \mathrm{C}\left([0, \infty) ; \mathrm{w}-\mathrm{L}^{2}(\Omega)\right) \cap \mathrm{L}_{\text {loc }}^{2}\left([0, \infty) ; \mathrm{L}^{2}(\Omega)\right),
$$

not necessarily unique, that satisfies the weak form of the system (1.1) given by

$$
\begin{gathered}
\left\langle\psi \omega\left(t_{2}\right)\right\rangle-\left\langle\psi \omega\left(t_{1}\right)\right\rangle-\int_{t_{1}}^{t_{2}}\langle(\boldsymbol{u} \cdot \nabla \psi) \omega\rangle \mathrm{d} t=0, \\
\boldsymbol{u}=K \omega, \\
\omega(0)=\omega^{\text {in }},
\end{gathered}
$$


for every $\left[t_{1}, t_{2}\right] \subset[0, \infty)$ and every test function $\psi \in \mathrm{H}_{0}^{1}(\Omega)$. It satisfies the "energy relation"

$$
\|\omega(t)\|_{\mathrm{L}^{2}} \leq\left\|\omega^{\mathrm{in}}\right\|_{\mathrm{L}^{2}},
$$

for every $t \in[0, \infty)$. Moreover, if $\omega^{\text {in }} \in \mathrm{L}^{\infty}(\Omega)$ then the weak form has a unique solution

$$
\omega \in \mathrm{C}\left([0, \infty) ; \mathrm{w}^{*}-\mathrm{L}^{\infty}([0, \infty) \times \Omega)\right) \cap \mathrm{L}^{\infty}([0, \infty) \times \Omega)
$$

that satisfies

$$
\|\omega(t)\|_{L^{\infty}} \leq\left\|\omega^{\text {in }}\right\|_{L^{\infty}}
$$

for every $t \in[0, \infty)$.

Remark 7 Given a topological vector space $\mathrm{X}$ with dual $\mathrm{X}^{\prime}$, a sequence of functions $\varphi_{\nu}:[0, \infty) \rightarrow \mathrm{X}^{\prime}$ converges to $\varphi$ in $\mathrm{C}\left(\left[0, \infty ; \mathrm{w}^{*}-\mathrm{X}^{\prime}\right)\right.$, where $\mathrm{w}^{*}-\mathrm{X}^{\prime}$ denotes $\mathrm{X}^{\prime}$ endowed with its weak-* topology, when for every $\psi \in \mathrm{X}$

$$
\left\langle\varphi_{\nu}(t), \psi\right\rangle_{\mathrm{X}^{\prime}, \mathrm{X}} \rightarrow\langle\varphi(t), \psi\rangle_{\mathrm{X}^{\prime}, \mathrm{X}}
$$

uniformly on compact subsets of $[0, \infty)$.

Proof. The proof will closely mirror the first four steps of the previous proof, albeit in weaker spaces. The proof will focus on the case of $\mathrm{L}^{\infty}$ initial data. The first statement of the theorem holds even for $\omega^{\text {in }} \in \mathrm{L}^{2}(\Omega)$, and its proof can be abstracted from Steps $1-4$ below by recasting appropriate $\mathrm{L}^{p}$ or $\mathrm{L}^{\infty}$ statements in $\mathrm{L}^{2}$.

Step 1 Use Theorem 6 to construct a family of approximate solutions

$$
\left\{\omega_{\nu}\right\} \subset \mathrm{C}\left([0, \infty) ; \mathrm{w}-\mathrm{H}_{0}^{1}(\Omega)\right) \cap \mathrm{L}_{\text {loc }}^{2}\left([0, \infty) ; \mathrm{H}^{2}(\Omega) \cap \mathrm{H}_{0}^{1}(\Omega)\right)
$$

that satisfy the weak form of the viscous equations (4.5), given by

$$
\begin{aligned}
&\left\langle\psi \omega_{\nu}\left(t_{2}\right)\right\rangle-\left\langle\psi \omega_{\nu}\left(t_{1}\right)\right\rangle+\nu \int_{t_{1}}^{t_{2}}\left\langle\nabla \psi \cdot \nabla \omega_{\nu}\right\rangle \mathrm{d} t \\
&-\int_{t_{1}}^{t_{2}}\left\langle\left(\boldsymbol{u}_{\nu} \cdot \nabla \psi\right) \omega_{\nu}\right\rangle \mathrm{d} t=0, \\
& \boldsymbol{u}_{\nu}=K \omega_{\nu}, \\
& \omega_{\nu}(0)=\omega_{\nu}^{\text {in }},
\end{aligned}
$$

for every $\left[t_{1}, t_{2}\right] \subset[0, \infty)$ and every test function $\psi \in \mathrm{H}_{0}^{1}(\Omega)$, as well as the "energy relation"

$$
\left\|\omega_{\nu}(t)\right\|_{\mathrm{L}^{p}} \leq\left\|\omega_{\nu}^{\mathrm{in}}\right\|_{\mathrm{L}^{p}},
$$

for every $t \in[0, \infty)$ and $2 \leq p<\infty$. 
The regularized initial data necessary for the application of Theorem 6 can for example be achieved by extending $\omega^{\text {in }}$ as zero outside

$$
\Omega_{2 \nu}=\{\boldsymbol{x} \in \Omega: \operatorname{dist}(\boldsymbol{x}, \partial \Omega)>2 \nu\},
$$

and setting

$$
\omega_{\nu}^{\text {in }} \equiv j_{\nu} *\left(\omega^{\text {in }} \chi\left(\Omega_{2 \nu}\right)\right) \in \mathrm{C}_{0}^{\infty}(\Omega),
$$

where $j_{\nu}$ is a Friedrichs mollifier. The regularized function $\omega_{\nu}^{\text {in }}$ converges to $\omega^{\text {in }}$ in $\mathrm{L}^{p}(\Omega)$ and satisfies $\left\|\omega_{\nu}^{\text {in }}\right\|_{\mathrm{L}^{p}} \leq\left\|\omega^{\text {in }}\right\|_{\mathrm{L}^{p}}$.

Step 2 Show that $\left\{\omega_{\nu}\right\}$ is relatively compact in $\mathrm{C}\left([0, \infty) ; \mathrm{w}-\mathrm{L}^{2}(\Omega)\right)$ and that for $\omega^{\text {in }} \in \mathrm{L}^{\infty}(\Omega),\left\{\omega_{\nu}\right\}$ is relatively compact in $\mathrm{C}\left([0, \infty)\right.$; $\left.\mathrm{w}^{*}-\mathrm{L}^{\infty}([0, \infty) \times \Omega)\right)$.

Proof of Step 2. We use the Arzela-Ascoli theorem as in the previous proof. Pointwise $\mathrm{L}^{2}$ bounds are guaranteed by (5.9) while the weak equicontinuity can be proved as in Step 2 of the previous proof - the estimates given there do not fail in the limit $\nu \rightarrow 0$. The Arzela-Ascoli theorem then assures that $\left\{\omega_{\nu}\right\}$ is a relatively compact set in $\mathrm{C}\left([0, \infty) ; \mathrm{w}-\mathrm{L}^{2}(\Omega)\right)$.

If moreover $\omega^{\text {in }} \in \mathrm{L}^{\infty}(\Omega)$, (5.9) holds uniformly for all $2 \leq p<\infty$, and hence

$$
\left\|\omega_{\nu}(t)\right\|_{\mathrm{L}^{\infty}} \leq\left\|\omega_{\nu}^{\mathrm{in}}\right\|_{\mathrm{L}^{\infty}} .
$$

This shows that $\left\{\omega_{\nu}(t)\right\}$ is a relatively compact set in $\mathrm{w}^{*}-\mathrm{L}^{\infty}([0, \infty) \times \Omega)$ for every $t \geq 0$. The statement is proved once we show that the sequence $\left\{\omega_{\nu}\right\}$ is equicontinuous in $\mathrm{C}\left([0, \infty)\right.$; $\left.\mathrm{w}^{*}-\mathrm{L}^{\infty}([0, \infty) \times \Omega)\right)$, i.e. that $\left\{\left\langle\psi \omega_{\nu}\right\rangle\right\}$ is equicontinuous in $C([0, \infty))$ for every $\psi \in \mathrm{L}^{1}(\Omega)$. This readily follows from the fact that $\mathrm{L}^{2}(\Omega) \hookrightarrow \mathrm{L}^{1}(\Omega)$ is dense plus the density argument given in Step 2 of the proof of Theorem 6 .

Step 3 Show that $\left\{\omega_{\nu}\right\}$ is a relatively compact set in $\mathrm{L}_{\text {loc }}^{2}\left([0, \infty) ; \mathrm{H}^{-1}(\Omega)\right)$.

Proof of Step 3. The statement follows from Step 2 and the continuity of the embeddings

$$
\begin{aligned}
\mathrm{C}\left([0, \infty) ; \mathrm{w}-\mathrm{L}^{2}(\Omega)\right) & \hookrightarrow \mathrm{C}\left([0, \infty) ; \mathrm{w}^{-} \mathrm{H}^{-1}(\Omega)\right) \wedge \mathrm{w}-\mathrm{L}_{\text {loc }}^{2}\left([0, \infty) ; \mathrm{w}-\mathrm{L}^{2}(\Omega)\right) \\
& \hookrightarrow \mathrm{L}_{\mathrm{loc}}^{2}\left([0, \infty) ; \mathrm{H}^{-1}(\Omega)\right)
\end{aligned}
$$

which are proved as in [8]. 
Step 4 Show that there is a subsequence of $\left\{\omega_{\nu}\right\}$ converging to a function $\omega$ which satisfies the weak form (1.9) and the energy relation (5.5).

Proof of Step 4. Steps 2 and 3 assert that there exists a subsequence of $\left\{\omega_{\nu}\right\}$ which we will still denote by $\left\{\omega_{\nu}\right\}$ that converges to a function

$$
\omega \in \mathrm{C}\left([0, \infty) ; \mathrm{w}^{*}-\mathrm{L}^{\infty}([0, \infty) \times \Omega)\right) \cap \mathrm{L}^{\infty}([0, \infty) \times \Omega)
$$

in the following sense:

(i) $\omega_{\nu} \rightarrow \omega$ in $\mathrm{C}\left([0, \infty) ; \mathrm{w}^{*}-\mathrm{L}^{\infty}(\Omega)\right)$;

(ii) $\omega_{\nu} \rightarrow \omega$ in $\mathrm{w}^{*}-\mathrm{L}^{\infty}([0, \infty) \times \Omega)$;

(iii) $\omega_{\nu} \rightarrow \omega$ in $\mathrm{L}_{\text {loc }}^{2}\left([0, \infty) ; \mathrm{H}^{-1}(\Omega)\right)$.

We claim that the regularized weak form (5.8a) converges to the weak form (5.2a) as $\nu \rightarrow 0$. We first restrict ourselves to test functions $\psi \in \mathrm{C}_{0}^{\infty}(\Omega)$ and then extend the result by density to all $\psi \in \mathrm{H}_{0}^{1}(\Omega)$.

Since (i) implies convergence in $\mathrm{C}\left([0, \infty)\right.$; $\left.-\mathrm{L}^{2}(\Omega)\right)$, we infer that

$$
\left\langle\psi \omega_{\nu}(t)\right\rangle \rightarrow\langle\psi \omega(t)\rangle,
$$

for every $t \geq 0$ as $\nu \rightarrow 0$. The viscosity term can be bounded as

$$
\nu \int_{t_{1}}^{t_{2}}\left\langle\nabla \psi \cdot \nabla \omega_{\nu}\right\rangle \mathrm{d} t \leq \nu\|\psi\|_{\mathrm{H}^{2}} \int_{t_{1}}^{t_{2}}\left\|\omega_{\nu}\right\|_{\mathrm{L}^{2}} \mathrm{~d} t .
$$

The last two factors on the right are bounded independent of $\nu$, so that the left side must converge to zero as $\nu \rightarrow 0$. To show the convergence of the nonlinear term we use the estimate

$$
\begin{aligned}
& \int_{t_{1}}^{t_{2}}\left(\left\langle\left(\boldsymbol{u}_{\nu} \cdot \nabla \psi\right) \omega_{\nu}\right\rangle-\langle(\boldsymbol{u} \cdot \nabla \psi) \omega\rangle\right) \mathrm{d} t \\
= & \int_{t_{1}}^{t_{2}}\left(\left\langle\left(\left(\boldsymbol{u}_{\nu}-\boldsymbol{u}\right) \cdot \nabla \psi\right) \omega_{\nu}\right\rangle+\left\langle(\boldsymbol{u} \cdot \nabla \psi)\left(\omega_{\nu}-\omega\right)\right\rangle\right) \mathrm{d} t \\
\leq & \int_{t_{1}}^{t_{2}}\left\|\boldsymbol{u}_{\nu}-\boldsymbol{u}\right\|_{\mathrm{L}^{2}}\|\nabla \psi\|_{\mathrm{L}^{\infty}}\left\|\omega_{\nu}\right\|_{\mathrm{L}^{2}} \mathrm{~d} t \\
& +\left|\int_{t_{1}}^{t_{2}}\left\langle(\boldsymbol{u} \cdot \boldsymbol{\nabla} \psi)\left(\omega_{\nu}-\omega\right)\right\rangle \mathrm{d} t\right| .
\end{aligned}
$$

Thus, in order to pass to the limit it is sufficient to have strong convergence of $\left\{\boldsymbol{u}_{\nu}\right\}$ in $\mathrm{L}_{\text {loc }}^{2}\left([0, \infty) ; \mathrm{L}^{2}\left(\Omega, \mathbb{R}^{2}\right)\right)$ and weak convergence of $\left\{\omega_{\nu}\right\}$ in $\mathrm{L}_{\text {loc }}^{2}\left([0, \infty) ; \mathrm{L}^{2}(\Omega)\right)$, properties that follow directly from (ii) and (iii). 
Finally, for $p<\infty$, the convergence of the energy relation (5.9) follows from the fact that $\omega_{\nu}^{\text {in }} \rightarrow \omega^{\text {in }}$ in $\mathrm{L}^{p}$ and that the convergence (i) implies

$$
\|\omega(t)\|_{\mathrm{L}^{p}} \leq \liminf _{\nu \rightarrow 0}\left\|\omega_{\nu}(t)\right\|_{\mathrm{L}^{p}},
$$

for every $t \geq 0$. As $\|\omega(t)\|_{\mathrm{L}^{p}} \leq\left\|\omega^{\text {in }}\right\|_{\mathrm{L}^{p}}$ uniformly over all $p<\infty$, it also holds for $p=\infty$, thus $\omega \in \mathrm{L}^{\infty}([0, \infty) \times \Omega)$.

Step 5 Show that the solution $\omega$ is unique.

Proof of Step 5. In order to prove uniqueness, we first need to recover the time derivative of $\omega$ in a weak sense. One has the estimate

$$
\int_{0}^{t}\left|\left\langle\left(\boldsymbol{u}\left(t^{\prime}\right) \cdot \nabla \psi\right) \omega\left(t^{\prime}\right)\right\rangle\right|^{2} \mathrm{~d} t^{\prime} \leq\|\psi\|_{\mathrm{H}^{1}}^{2} \int_{0}^{t}\left\|\boldsymbol{u}\left(t^{\prime}\right)\right\|_{\mathrm{L}^{4}}^{2}\left\|\omega\left(t^{\prime}\right)\right\|_{\mathrm{L}^{4}}^{2} \mathrm{~d} t^{\prime},
$$

and $\omega \in \mathrm{L}^{\infty}([0, \infty) \times \Omega)$, so that the integral on the right is finite. We conclude that the mapping $(t, \psi) \mapsto\langle(\boldsymbol{u}(t) \cdot \nabla \psi) \omega(t)\rangle$ defines a function in $\mathrm{L}_{\text {loc }}^{2}\left([0, \infty) ; \mathrm{H}^{-1}(\Omega)\right)$.

Let therefore $\psi \in \mathrm{H}_{0}^{1}(\Omega)$. The weak form (5.2a) includes in particular

$$
\langle\psi \omega(t)\rangle=\left\langle\psi \omega^{\text {in }}\right\rangle+\int_{0}^{t}\langle(\boldsymbol{u} \cdot \boldsymbol{\nabla} \psi) \omega\rangle \mathrm{d} t^{\prime} .
$$

Take a test function $\theta \in C_{0}^{\infty}([0, \infty))$, multiply the previous line with $\mathrm{d} \theta / \mathrm{d} t$, integrate from 0 to $\infty$ and then integrate by parts to obtain

$$
\int_{0}^{\infty}\langle\psi \omega(t)\rangle \frac{\mathrm{d} \theta}{\mathrm{d} t}(t) \mathrm{d} t=\int_{0}^{\infty}\langle(\boldsymbol{u}(t) \cdot \nabla \psi) \omega(t)\rangle \theta(t) \mathrm{d} t .
$$

Integration by parts is legitimate here because from our earlier remark it follows that the integrand on the right side of $(5.20)$ is a $\mathrm{L}_{\text {loc }}^{1}([0, \infty))$ function. Relation (5.21) is equivalent to saying that

$$
\frac{\mathrm{d}}{\mathrm{d} t}\langle\psi \omega(t)\rangle=\langle(\boldsymbol{u}(t) \cdot \nabla \psi) \omega(t)\rangle
$$

in the sense of distributions on $[0, \infty)$. Even more, we can write

$$
\partial_{t} \omega=-b^{-1} \boldsymbol{\nabla} \cdot(b \boldsymbol{u} \omega) \in \mathrm{L}_{\mathrm{loc}}^{2}\left([0, \infty) ; \mathrm{H}^{-1}(\Omega)\right) .
$$

We also recall that $\omega \in \mathrm{L}_{\text {loc }}^{2}\left([0, \infty) ; \mathrm{L}^{2}(\Omega)\right)$. It follows that the following identity holds in the sense of distributions on $\mathbb{R}$ :

$$
\frac{1}{2} \frac{\mathrm{d}}{\mathrm{d} t}\|\omega(t)\|_{\mathrm{H}^{-1}}^{2}=\left(\partial_{t} \omega, \omega\right)_{\mathrm{H}^{-1}} .
$$


This identity is obvious for functions $\omega \in \mathrm{C}_{0}^{\infty}\left(\mathbb{R} ; \mathrm{H}^{-1}(\Omega)\right)$ and can be extended by continuity to all $\omega \in \mathrm{L}_{\text {loc }}^{2}\left([0, \infty) ; \mathrm{H}^{-1}(\Omega)\right)$ with $\partial_{t} \omega \in \mathrm{L}_{\text {loc }}^{2}\left([0, \infty) ; \mathrm{H}^{-1}(\Omega)\right)$ by a density argument. It then follows that $\omega \in \mathrm{C}\left([0, \infty) ; \mathrm{H}^{-1}(\Omega)\right)$, see Wloka $[21$, Theorem 25.5].

Now assume that $\hat{\omega}$ and $\bar{\omega}$ are two solutions to equation (5.23) that correspond to identical initial data and set $\hat{\boldsymbol{u}}=K \hat{\omega}, \overline{\boldsymbol{u}}=K \overline{\boldsymbol{\omega}}$ and $\varphi=\hat{\omega}-\bar{\omega}$. Our goal is to compute the time evolution of $\varphi$ in the $\mathrm{H}^{-1}$ norm. If we can show that this norm remains zero for all times, the two solutions must be identical. Note that this does not mean that we allow general $\mathrm{H}^{-1}$ initial data for $\hat{\omega}$ or $\bar{\omega}$.

First observe that for any two functions $\boldsymbol{v} \in \mathrm{L}^{2}\left(\Omega, \mathbb{R}^{2}\right)$ and $\psi \in \mathrm{L}^{2}(\Omega)$ one has

$$
\left\langle A^{-1}\left(b^{-1} \nabla \cdot(b \boldsymbol{v})\right) \psi\right\rangle=-\left\langle\boldsymbol{v} \cdot \nabla A^{-1} \psi\right\rangle .
$$

This is easily verified for smooth functions and extends by continuity. Using (5.24) and then (5.25),

$$
\begin{aligned}
\frac{1}{2} \frac{\mathrm{d}}{\mathrm{d} t}\|\varphi(t)\|_{\mathrm{H}^{-1}}^{2} & =-\left\langle A^{-1}\left(b^{-1} \nabla \cdot(b \hat{\boldsymbol{u}} \hat{\omega})\right) \varphi\right\rangle+\left\langle A^{-1}\left(b^{-1} \nabla \cdot(b \overline{\boldsymbol{u}} \bar{\omega})\right) \varphi\right\rangle \\
& =\left\langle\hat{\omega} \hat{\boldsymbol{u}} \cdot \nabla A^{-1} \varphi\right\rangle-\left\langle\bar{\omega} \overline{\boldsymbol{u}} \cdot \nabla A^{-1} \varphi\right\rangle \\
& =\left\langle\varphi \hat{\boldsymbol{u}} \cdot \nabla A^{-1} \varphi\right\rangle+\left\langle\bar{\omega}(\hat{\boldsymbol{u}}-\overline{\boldsymbol{u}}) \cdot \nabla A^{-1} \varphi\right\rangle .
\end{aligned}
$$

The second term on the right is estimated

$$
\left|\left\langle\bar{\omega}(\hat{\boldsymbol{u}}-\overline{\boldsymbol{u}}) \cdot \nabla A^{-1} \varphi\right\rangle\right| \leq\|\bar{\omega}\|_{\mathrm{L}^{\infty}}\|\hat{\boldsymbol{u}}-\overline{\boldsymbol{u}}\|_{\mathrm{L}^{2}}\|\varphi\|_{\mathrm{H}^{-1}} \leq c_{1}\|\varphi\|_{\mathrm{H}^{-1}}^{2},
$$

using the global $\mathrm{L}^{\infty}$ bound on $\bar{\omega}$ and the continuity of $K$. The first term on the right of (5.26) is estimated by noting that for every $\boldsymbol{u} \in \mathbb{D}$ and $\psi \in \mathrm{C}_{0}^{\infty}(\Omega)$

$$
\begin{aligned}
\langle A(\boldsymbol{u} \cdot \boldsymbol{\nabla} \psi) \psi\rangle & =\langle\boldsymbol{\nabla}(\boldsymbol{u} \cdot \boldsymbol{\nabla} \psi) \cdot \boldsymbol{\nabla} \psi\rangle \\
& =\langle\boldsymbol{\nabla} \psi \cdot(\boldsymbol{\nabla} \boldsymbol{u}) \cdot \boldsymbol{\nabla} \psi\rangle+\langle\boldsymbol{u} \cdot(\boldsymbol{\nabla} \nabla \psi) \cdot \boldsymbol{\nabla} \psi\rangle .
\end{aligned}
$$

The second term on the right vanishes due to relation (4.4). Then, by taking $\psi=A^{-1} \varphi$, still assuming classical functions,

$$
\begin{aligned}
\left|\left\langle\varphi \boldsymbol{u} \cdot \nabla A^{-1} \varphi\right\rangle\right| & =|\langle\psi A(\boldsymbol{u} \cdot \nabla \psi)\rangle|=|\langle\boldsymbol{\nabla} \psi \cdot(\boldsymbol{\nabla} \boldsymbol{u}) \cdot \boldsymbol{\nabla} \psi\rangle| \\
& \leq\|\boldsymbol{u}\|_{\mathrm{W}^{1, p}}\|\psi\|_{\mathrm{W}^{1,2 q}}^{2}, \quad \text { where } \frac{1}{p}+\frac{1}{q}=1 .
\end{aligned}
$$

This estimate extends by continuity to all $\boldsymbol{u} \in \mathrm{W}^{1, p}\left(\Omega, \mathbb{R}^{2}\right)$ with $\boldsymbol{\nabla} \cdot(b \boldsymbol{u})=0$ on $\Omega$ and $\boldsymbol{u} \cdot \boldsymbol{n}=0$ on $\partial \Omega$, and all $\varphi \in \mathrm{L}^{2}(\Omega)$. Then

$$
\begin{aligned}
\left|\left\langle\varphi \hat{\boldsymbol{u}} \cdot \nabla A^{-1} \varphi\right\rangle\right| & \leq\|\hat{\boldsymbol{u}}\|_{\mathrm{W}^{1, p}}\left\|A^{-1} \varphi\right\|_{\mathrm{W}^{1,2 q}}^{2} \\
& \leq p c_{2}\left\|A^{-1 / 2} \varphi\right\|_{\mathrm{L}^{2 q}}^{2} \\
& \leq p c_{3}\|\varphi\|_{\mathrm{H}^{-1}}^{2-2 / p} .
\end{aligned}
$$


The last step is valid because $\varphi \in \mathrm{L}^{\infty}([0, \infty) \times \Omega)$ implies that $A^{-1 / 2} \varphi \in$ $\mathrm{L}^{\infty}([0, \infty) \times \Omega)$. Altogether one obtains

$$
\frac{\mathrm{d}}{\mathrm{d} t}\|\varphi(t)\|_{\mathrm{H}^{-1}}^{2} \leq p c_{4}\|\varphi(t)\|_{\mathrm{H}^{-1}}^{2-2 / p} .
$$

Since $\|\varphi(0)\|_{\mathrm{H}^{-1}}^{2}=0$, we have in particular that $\|\varphi(0)\|_{\mathrm{H}^{-1}}^{2} \leq 2^{-p}$ for every $p$. This allows us to integrate this differential inequality away from the singularity when $\|\varphi(t)\|_{\mathrm{H}^{-1}}^{2}=0$ and we obtain the bound

$$
\|\varphi(t)\|_{\mathrm{H}^{-1}}^{2} \leq\left(\frac{1}{2}+c_{4} t\right)^{p}
$$

Taking the limit $p \rightarrow \infty$ shows that $\varphi(t)=0$ on the interval $\left[0, t_{0}\right]$ whenever $\frac{1}{2}+c_{4} t_{0}<1$. Tiling the half line $[0, \infty)$ with intervals of length $t_{0}$ and repeating the argument then shows that $\varphi(t)=0$ for every $t \geq 0$. This completes the proof of Theorem 7 .

Remark 8 The above proof of uniqueness shows how one can work around an inconsistency in the original proof given by Bardos [4]. Another way to do so has been pointed out by Kato [12].

Remark 9 The abstract result, Theorem 7, can actually be proved under the weaker assumption $\partial \Omega \in \mathrm{C}^{2}$ as stated in Theorem 1 by regularizing not only the initial data, but also the boundary before applying Theorem 6 . Similarly, the continuity requirements on $K$ can be weakened in the inviscid limit to continuity of $K: \mathrm{L}^{2}(\Omega) \rightarrow \mathbb{V}$ only. This would reduce the continuity requirements on $b$ and $\partial \Omega$ in Lemma 3 by one order, but burden the proof of Theorem 7 with cumbersome technicalities.

6. Continuous dependence on the data. Now we estimate how the solutions depend on bottom topography and initial data. The strategy is very similar to that of the uniqueness argument in the previous section. Again we consider two solutions $\hat{\omega}$ and $\bar{\omega}$ to the lake equations, but with bottom functions $\hat{b}$ and $\bar{b}$ respectively. Their difference $\varphi=\hat{\omega}-\bar{\omega}$ is then estimated as before, with some additional terms arising due to the difference in the bottom topographies. All quantities associated with bottom topography $\hat{b}$ will also carry the hat. For example, we will denote by $\hat{\mathrm{H}}^{n}$ the $\mathrm{H}^{n}$ spaces with weight $\hat{b}$. Analogously, quantities associated with $\bar{b}$ will carry the bar.

An estimate on the time evolution of the $\overline{\mathrm{H}}^{-1}$ norm can be obtained in a way similar to the derivation of (5.26). Note that (5.25) holds whether or not 
$\boldsymbol{\nabla} \cdot(b \boldsymbol{v})=0$. A direct calculation using (5.25) gives

$$
\begin{aligned}
& \frac{1}{2} \frac{\mathrm{d}}{\mathrm{d} t}\|\varphi(t)\|_{\overline{\mathrm{H}}^{-1}}^{2}=-\left\langle\bar{A}^{-1}\left(\hat{b}^{-1} \boldsymbol{\nabla} \cdot(\hat{b} \hat{\boldsymbol{u}} \hat{\omega})\right) \varphi \overline{\rangle}+\left\langle\bar{A}^{-1}\left(\bar{b}^{-1} \boldsymbol{\nabla} \cdot(\bar{b} \overline{\boldsymbol{u}} \bar{\omega})\right) \varphi \overline{\rangle}\right.\right. \\
& =\left\langle\hat{\omega} \hat{\boldsymbol{u}} \cdot \nabla\left(\bar{b}^{-1} \bar{A}^{-1} \varphi\right) \hat{\rangle}-\left\langle\bar{\omega} \overline{\boldsymbol{u}} \cdot \nabla \bar{A}^{-1} \varphi \overline{\rangle}\right.\right. \\
& =\left\langle\hat{\omega} \hat{\boldsymbol{u}} \cdot \nabla\left(\bar{b}\left(\hat{b}^{-1}-\bar{b}^{-1}\right) \bar{A}^{-1} \varphi\right) \hat{\rangle}+\int_{\Omega} \hat{\omega}\left(\hat{\boldsymbol{u}} \cdot \nabla \bar{A}^{-1} \varphi\right)(\hat{b}-\bar{b}) \mathrm{d}^{2} \boldsymbol{x}\right. \\
& +\left\langle\hat{\omega}(\hat{\boldsymbol{u}}-\overline{\boldsymbol{u}}) \cdot \nabla \bar{A}^{-1} \varphi \overline{\rangle}+\left\langle\varphi \overline{\boldsymbol{u}} \cdot \nabla \bar{A}^{-1} \varphi \overline{\rangle}\right.\right. \\
& \equiv I_{1}+I_{2}+I_{3}+I_{4} \text {. }
\end{aligned}
$$

We now derive bounds on the integrals $I_{1}-I_{4}$. The first two can be estimated directly by Hölder inequalities. We employ the same strategy as in the derivation of (5.30), so that for $\frac{1}{p}+\frac{1}{q}=1$ one has

$$
\begin{aligned}
\left|I_{1}\right| & \leq c\|\hat{\omega}\|_{\mathrm{L}^{\infty}}\|\hat{\boldsymbol{u}}\|_{\mathrm{L}^{p}}\left\|\hat{b}^{-1}-\bar{b}^{-1}\right\|_{\mathrm{W}^{1,2 q}}\left\|\bar{A}^{-1} \varphi\right\|_{\mathrm{W}^{1,2 q}} \\
& \leq c p\|\hat{b}-\bar{b}\|_{\mathrm{W}^{1,2 q}}\|\varphi\|_{\overline{\mathrm{H}}^{-1}}^{1-1 / p}
\end{aligned}
$$

and similarly,

$$
\left|I_{2}\right| \leq c p\|\hat{b}-\bar{b}\|_{\mathrm{L}^{2 q}}\|\varphi\|_{\overline{\mathrm{H}}^{-1}}^{1-1 / p}
$$

In order to bound $I_{3}$ we first estimate

$$
\left|I_{3}\right| \leq c\|\hat{\omega}\|_{L^{\infty}}\|\hat{\boldsymbol{u}}-\overline{\boldsymbol{u}}\|_{\overline{\mathrm{L}}^{2}}\left\|\bar{A}^{-1} \varphi\right\|_{\overline{\mathrm{H}}^{1}} .
$$

Here the $\overline{\mathrm{L}}^{2}$ difference of $\hat{\boldsymbol{u}}$ and $\overline{\boldsymbol{u}}$ requires a more subtle analysis than in the corresponding estimate of the uniqueness argument, equation (5.27). Recall that $\bar{P}$ denotes the $\overline{\mathrm{L}}^{2}$-orthogonal projector onto $\overline{\mathbb{H}}$, so that we can write

$$
\begin{aligned}
\hat{\boldsymbol{u}}-\overline{\boldsymbol{u}} & =\left(1-\bar{K} \hat{K}^{-1}\right) \hat{\boldsymbol{u}}+\bar{K}(\hat{\omega}-\bar{\omega}) \\
& =\bar{K}\left(\bar{K}^{-1} \bar{P}-\hat{K}^{-1} \hat{P}\right) \hat{\boldsymbol{u}}+\bar{Q} \hat{\boldsymbol{u}}+\bar{K} \varphi .
\end{aligned}
$$

This form is particularly useful because Lemma 2 gives an explicit expression for $K^{-1} P$ which, even in the case of the great lake equations does not loose more that one derivative. Observe that $\mathbb{H}^{\perp}=Q L^{2}\left(\Omega, \mathbb{R}^{2}\right)$ is contained in the null space of the curl operator, so that

$$
\begin{aligned}
K^{-1} P \boldsymbol{u} \equiv b^{-1} \nabla \wedge \mathcal{L} P \boldsymbol{u} & =b^{-1} \nabla \wedge P \mathcal{L} P \boldsymbol{u} \\
& =b^{-1} \nabla \wedge\left(\boldsymbol{u}+\frac{\delta^{2}}{3} \nabla b(\boldsymbol{\nabla} b \cdot P \boldsymbol{u})\right),
\end{aligned}
$$

for every $\boldsymbol{u} \in \mathrm{L}^{2}\left(\Omega, \mathbb{R}^{2}\right)$. Using this identity with bottom functions $\hat{b}$ and $\bar{b}$ 
respectively, it is easy to rewrite

$$
\begin{aligned}
\left(\bar{K}^{-1}\right. & \left.\bar{P}-\hat{K}^{-1} \hat{P}\right) \hat{\boldsymbol{u}} \\
=\bar{b}^{-1} \boldsymbol{\nabla} & \wedge\left(\hat{\boldsymbol{u}}+\frac{\delta^{2}}{3} \boldsymbol{\nabla} \bar{b}(\boldsymbol{\nabla} \bar{b} \cdot \bar{P} \hat{\boldsymbol{u}})\right)-\hat{b}^{-1} \boldsymbol{\nabla} \wedge\left(\hat{\boldsymbol{u}}+\frac{\delta^{2}}{3} \boldsymbol{\nabla} \hat{b}(\boldsymbol{\nabla} \hat{b} \cdot \hat{\boldsymbol{u}})\right) \\
=\left(\bar{b}^{-1}-\right. & \left.\hat{b}^{-1}\right) \boldsymbol{\nabla} \wedge \hat{\boldsymbol{u}}+\frac{\delta^{2}}{3}\left[\bar{b}^{-1} \boldsymbol{\nabla} \wedge(\boldsymbol{\nabla} \bar{b}(\boldsymbol{\nabla} \bar{b} \cdot(\bar{P}-1) \hat{\boldsymbol{u}}))\right. \\
& +\bar{b}^{-1} \boldsymbol{\nabla} \wedge(\boldsymbol{\nabla} \bar{b}(\boldsymbol{\nabla}(\bar{b}-\hat{b}) \cdot \hat{\boldsymbol{u}})) \\
& +\bar{b}^{-1} \boldsymbol{\nabla} \wedge(\boldsymbol{\nabla}(\bar{b}-\hat{b})(\boldsymbol{\nabla} \hat{b} \cdot \hat{\boldsymbol{u}})) \\
& \left.+\left(\bar{b}^{-1}-\hat{b}^{-1}\right) \boldsymbol{\nabla} \wedge(\boldsymbol{\nabla} \hat{b}(\boldsymbol{\nabla} \hat{b} \cdot \hat{\boldsymbol{u}}))\right] .
\end{aligned}
$$

Hence,

$$
\begin{aligned}
& \left\|\bar{K}\left(\bar{K}^{-1} \bar{P}-\hat{K}^{-1} \hat{P}\right) \hat{\boldsymbol{u}}\right\|_{\mathrm{L}^{2}} \\
& \leq c\left\|\left(\bar{b}^{-1}-\hat{b}^{-1}\right) \boldsymbol{\nabla} \wedge \hat{\boldsymbol{u}}\right\|_{\mathrm{L}^{2}} \\
& \quad+c \delta^{2}\left[\|\boldsymbol{\nabla} \bar{b}(\boldsymbol{\nabla} \bar{b} \cdot(\bar{P}-1) \hat{\boldsymbol{u}})\|_{\mathrm{L}^{2}}+\|\boldsymbol{\nabla} \bar{b}(\boldsymbol{\nabla}(\bar{b}-\hat{b}) \cdot \hat{\boldsymbol{u}})\|_{\mathrm{L}^{2}}\right. \\
& \left.\quad+\|\boldsymbol{\nabla}(\bar{b}-\hat{b})(\boldsymbol{\nabla} \hat{b} \cdot \hat{\boldsymbol{u}})\|_{\mathrm{L}^{2}}+\left\|\left(\bar{b}^{-1}-\hat{b}^{-1}\right) \boldsymbol{\nabla} \wedge(\boldsymbol{\nabla} \hat{b}(\boldsymbol{\nabla} \hat{b} \cdot \hat{\boldsymbol{u}}))\right\|_{\mathrm{L}^{2}}\right] \\
& \leq c\|\hat{b}-\bar{b}\|_{\mathrm{L}^{2 q}}\|\hat{\boldsymbol{u}}\|_{\mathrm{W}^{1,2 p}}+c \delta^{2}\left[\|\bar{Q} \hat{\boldsymbol{u}}\|_{\mathrm{L}^{2}}+\|\hat{b}-\bar{b}\|_{\mathrm{W}^{1,2 q}}\|\hat{\boldsymbol{u}}\|_{\mathrm{W}^{1,2 p}}\right. \\
& \left.\quad+\|\hat{b}-\bar{b}\|_{\mathrm{L}^{2 q}}\|\hat{\boldsymbol{u}}\|_{\mathrm{W}^{1,2 p}}\right] \\
& \leq c p\|\hat{b}-\bar{b}\|_{\mathrm{W}^{1,2 q}}+c \delta^{2}\|\bar{Q} \hat{\boldsymbol{u}}\|_{\mathrm{L}^{2}} .
\end{aligned}
$$

In this estimate we have extensively used the fact that $b \in \mathrm{C}^{2}(\bar{\Omega})$. An estimate on $\bar{Q} \hat{\boldsymbol{u}}$ is obtained as follows. The projection is computed by solving the Neumann problem

$$
\begin{gathered}
\bar{b}^{-1} \boldsymbol{\nabla} \cdot(\bar{b} \hat{\boldsymbol{u}})=-\bar{A} \psi \quad \text { in } \Omega, \\
\boldsymbol{n} \cdot \boldsymbol{\nabla} \psi=\boldsymbol{n} \cdot \overline{\boldsymbol{u}} \quad \text { on } \partial \Omega,
\end{gathered}
$$

and setting $\bar{Q} \hat{\boldsymbol{u}} \equiv \boldsymbol{\nabla} \psi$. Then, invoking elliptic regularity, one estimates

$$
\begin{aligned}
\|\bar{Q} \hat{\boldsymbol{u}}\|_{\overline{\mathrm{L}}^{2}} \leq\|\psi\|_{\overline{\mathrm{H}}^{1}} & \leq c\|\boldsymbol{\nabla} \cdot((\hat{b}-\bar{b}) \hat{\boldsymbol{u}})\|_{\overline{\mathrm{H}}^{-1}} \\
& \leq c\|\hat{b}-\bar{b}\|_{\mathrm{L}^{2 q}}\|\hat{\boldsymbol{u}}\|_{\mathrm{L}^{2 p}} \leq c p\|\hat{b}-\bar{b}\|_{\mathrm{L}^{2 q}} .
\end{aligned}
$$

Altogether, we obtain the following bound:

$$
\begin{aligned}
\left|I_{3}\right| & \leq c\|\hat{\boldsymbol{u}}-\overline{\boldsymbol{u}}\|_{\overline{\mathrm{L}}^{2}}\|\varphi\|_{\overline{\mathrm{H}}^{-1}} \\
& \leq c\left(\left\|\bar{K}\left(\bar{K} \bar{K}^{-1} \bar{P}-\hat{K}^{-1} \hat{P}\right) \hat{\boldsymbol{u}}\right\|_{\overline{\mathrm{L}}^{2}}+\|\bar{Q} \hat{\boldsymbol{u}}\|_{\overline{\mathrm{L}}^{2}}+\|\bar{K} \varphi\|_{\overline{\mathrm{L}}^{2}}\right)\|\varphi\|_{\overline{\mathrm{H}}^{-1}} \\
& \leq c p\|\hat{b}-\bar{b}\|_{\mathrm{L}^{2 q}}\|\varphi\|_{\overline{\mathrm{H}}^{-1}}+c\|\varphi\|_{\overline{\mathrm{H}}^{-1}}^{2} .
\end{aligned}
$$


Finally, $I_{4}$ is estimated by proceeding as in (5.29), namely

$$
\begin{aligned}
\left|I_{4}\right| & =\mid\left\langle\varphi \overline{\boldsymbol{u}} \cdot \nabla \bar{A}^{-1} \varphi \overline{\rangle}|=|\left\langle\left(\nabla \bar{A}^{-1} \varphi\right) \cdot(\nabla \overline{\boldsymbol{u}}) \cdot\left(\nabla \bar{A}^{-1} \varphi\right) \overline{\rangle}\right|\right. \\
& \leq\|\overline{\boldsymbol{u}}\|_{\mathrm{W}^{1, p}}\left\|\bar{A}^{-1} \varphi\right\|_{\mathrm{W}^{1,2 q}}^{2} \\
& \leq c p\left\|\bar{A}^{-1 / 2} \varphi\right\|_{\mathrm{L}^{2 q}}^{2} \leq c p\|\varphi\|_{\overline{\mathrm{H}}^{-1}}^{2-2 / p} .
\end{aligned}
$$

One can combine all these estimates for example, by using the $\mathrm{L}^{\infty}$ bound on $\varphi$ again, into the differential inequality

$$
\frac{\mathrm{d}}{\mathrm{d} t}\|\varphi(t)\|_{\overline{\mathrm{H}}^{-1}}^{2} \leq c p\left(\|\hat{b}-\bar{b}\|_{\mathrm{W}^{1,2 q}}+\|\varphi\|_{\overline{\mathrm{H}}^{-1}}\right)\|\varphi\|_{\overline{\mathrm{H}}^{-1}}^{1-2 / p} .
$$

Comparing this differential inequality with the corresponding inequality (5.31) in the proof of uniqueness, we see that the inequalities have the same structure, albeit here we have an extra driving term depending on the difference of the the bottom topographies. For $\varphi$ smaller in magnitude than the $\mathrm{L}^{\infty}$ bound on $\omega$ guaranteed by the existence result, Theorem 7 - which is the range of $\varphi$ for which the new estimate might be of any interest - the two solutions $\hat{\omega}$ and $\bar{\omega}$ drift apart marginally worse than exponentially in $\mathrm{H}^{-1}$. The fact that this behavior is intrinsic in both (5.31) and (6.13) suggests that it is the optimum obtainable by Sobolev estimation techniques. Lastly we remark that $\mathrm{H}^{-1}$ estimates on differences of the potential vorticities imply $L^{2}$ bounds on the velocity fields.

The foremost consequence of (6.13) is the continuous dependence of the solution at a fixed time $T$ on the bottom topography and the initial data. This can be seen as follows: given (6.13) with initial condition $\|\varphi(0)\|_{\overline{\mathrm{H}}^{-1}}$, the solution of

$$
\begin{gathered}
\frac{\mathrm{d} F}{\mathrm{~d} t}=c p F^{1-1 / p} \\
F(0)=\left(\|\hat{b}-\bar{b}\|_{\mathrm{W}^{1,2 q}}+\|\varphi(0)\|_{\overline{\mathrm{H}}^{-1}}\right)^{p /(p-1)}\|\varphi(0)\|_{\overline{\mathrm{H}}^{-1}}^{(p-2) /(p-1)},
\end{gathered}
$$

provides an upper bound on $\|\varphi(t)\|_{\overline{\mathrm{H}}^{-1}}$ for all times $t \geq 0$. This equation is easily solved, namely

$$
F(t)=\left(F^{1 / p}(0)+c t\right)^{p} .
$$

Now let $\varepsilon>0$. Then there exists a $p$ such that for every $F(0) \leq \delta \equiv 3^{-p}$ and $t \leq 1 /(3 c)$ one has $F(t)<\varepsilon$. Together with (6.14), this proves continuous dependence of $\omega$ in $\mathrm{H}^{-1}(\Omega)$ on $b$ in $\mathrm{W}^{1,2 q}(\Omega)$ and $\omega^{\text {in }}$ in $\mathrm{H}^{-1}(\Omega)$ on the interval $[0,1 /(3 c)]$. For arbitrary times $T$ one can tile the time axis with intervals of length $1 /(3 c)$ and iterate the argument. Thus we have the following.

Theorem 8 For every fixed time $T$ and every $q>1$, the mapping $\left(b, \omega^{\text {in }}\right) \mapsto$ $\omega(T)$ is continuous on $\mathrm{W}^{1,2 q}(\Omega) \times \mathrm{H}^{-1}(\Omega) \rightarrow \mathrm{H}^{-1}(\Omega)$. 
7. Acknowledgments. We thank R. Camassa, C. Doering, D. Holm and L. Margolin for interesting and stimulating discussions. Part of this work was done when the authors were visitors of the CNLS and the IGPP at Los Alamos National Laboratory. The first two authors would also like to thank the Ecole Normale Supérieure de Cachan for their hospitality during the finalization of this manuscript. This work was supported in part by the NSF grants No. DMS9308774 and DMS-9404570 and by the Joint University of California and Los Alamos National Laboratory INCOR program. E. S. Titi also acknowledges the kind hospitality of the Newton Institute, the partial support of the EPSRC Grant No. GR J82041 and the University of California-Irvine Faculty Research Fellowship.

\section{REFERENCES}

[1] R.S. Adams, Sobolev Spaces, Academic Press, New York, 1975.

[2] S. Agmon, A. Douglis and L. Nirenberg, Estimates near the Boundary for Solutions of Elliptic Partial Differential Equations I and II, Comm. Pure Appl. Math. 12 (1959), 623-727; 17 (1964), 35-92.

[3] V. Barcilon, P. Constantin And E.S. Titi, Existence of solutions to the StommelCharney model of the Gulf Stream, SIAM J. Math. Anal. 19 (1988), 1355-1364.

[4] C. Bardos, Existence et unicité de la solution de l'équation d'Euler en dimension deux, J. Math. Anal. and Appl. 40 (1972), 769-790.

[5] R. Camassa, D.D. Holm and C.D. Levermore, Long-Time Shallow Water Equations with a Varying Bottom, J. Fluid Mech. (submitted 1994).

[6] R. Camassa, D.D. Holm And C.D. Levermore, Long-Time Effects of Bottom Topograghy in Shallow Water, Physica D, (to appear 1996).

[7] P. Constantin and C. Foias, Navier-Stokes Equations, The University of Chicago Press, 1988.

[8] C.R. Doering, J.D. Gibbon And C.D. Levermore, Weak and Strong Solutions of the Complex Ginzburg-Landau Equation, Physica D 71 (1994), 285-318.

[9] M. Feistauer, Mathematical Methods in Fluid Dynamics, Wiley, New York, 1993.

[10] G.P. GALDI, An Introduction to the Mathematical Theory of the Navier-Stokes Equations, Springer-Verlag, New York, 1994.

[11] D. Gilbarg And N.S. Trudinger, Elliptic Partial Differential Equations of Second Order, Springer-Verlag, New York, 1983.

[12] T. Kato, A Remark on a Theorem of C. Bardos on the 2D-Euler Equation, Department of Mathematics, University of California at Berkeley, preprint (1992).

[13] C.D. Levermore, M. Oliver And E.S. Titi, Global Well-Posedness for the Lake Equations, Physica D (to appear 1996).

[14] C.D. Levermore And M. Oliver, Analyticity of Solutions for a Generalized Euler Equation, J. Diff. Eq. (to appear).

[15] C. Marchioro and M. Pulvirenti, Mathematical Theory of Incompressible Non-viscous Fluids, Springer-Verlag, New York, 1994.

[16] M. Oliver, PhD thesis, University of Arizona, 1996.

[17] M. Oliver, Classical Solutions for a Generalized Euler Equation in Two Dimensions, Comm. in P.D.E. (submitted 1996).

[18] J. Pedlosky, Geophysical Fluid Dynamics, Springer-Verlag, New York, 1987.

[19] R. Temam, Navier-Stokes Equations: Theory and Numerical Analysis, North-Holland, Amsterdam, 1984. 
[20] R. Temam, Infinite-Dimensional Dynamical Systems in Mechanics and Physics, Springer-Verlag, New York, 1988.

[21] J. Wloka, Partial Differential Equations, Cambridge University Press, 1987.

[22] V.I. Yudovitch, Non-stationary flow of an ideal incompressible liquid, Zh. Vychisl. Mat. i Mat. Fiz. 6 (1963), 1032-1066.

C. D. Levermore

Department of Mathematics

University of Arizona

Tucson, Arizona 85721

E-MAIL: lvrmr@math.arizona.edu

M. Oliver

Program in Applied Mathematics

University of Arizona

Tucson, Arizona 85721

E-MAIL: oliver@math.arizona.edu

\section{E.S. Titi}

Department of Mathematics and Department of Mechanical and Aerospace Engineering University of California

Irvine, California 92717

E-MAIL: etiti@math.uci.edu

Received: April 4th, 1996. 